\title{
Regular Solutions for the relativistic Boltzmann equation in Yang-Mills field
}

\section{Solutions régulières pour l'équation relativiste de Boltzmann dans le domaine de Yang-Mills}

\section{Dongo David and Nguelemo Kenfack Abel}

Department of Mathematics and computer science, Faculty of Science,

University of Dschang, POB : 67 Dschang, Cameroon

dongodavid@yahoo.fr, abeldecauchy@yahoo.fr

ABSTRACT. We consider in this work the Boltzmann equation in the presence of a Yang-Mills fields in temporal gauge, which generalizes to the non-Abelian case the electromagnetic field. We prove, using the method presented by N. Noutchegueme and R. D. Ayissi [2], a local in time existence and uniqueness theorem for the regular solutions.

KEYWORDS. Relativistic Boltzmann equation, charged particles, Yang-Mills field, regular solution, existence and uniqueness.

\section{Introduction}

A plasma is a collection of particles moving at very high speed and under the effect of the forces they create. For the electrons, in the Abelian case, the forces created are electromagnetic forces and when we consider the particles evolving with collisions, the self-mained phenomenon is governed by the Maxwell-Boltzmann system. Many authors have studied the relativistic Boltzmann equation alone (which is one of the basic equations of the relativistic Kinetic theory) or this equation coupled with the Maxwell equations : For the non classical solution, we can refer to [9], [13] and [14]. For the regular solutions, we can refer to [2], [10], [12] and references therein.

In this work, we consider a more general plasma with particles having non-Abelian charges, for example the field of quarks and gluons that one meets in chromodynamics, the electromagnetic field is replaced by the Yang-Mills field. The plasma obtained here, called "plasma quarks-gluons", is supposed to exist at very high temperature. In the collisionless case where the Boltzmann equation is replaced by the Vlasov equation, many authors have already studied this kind of phenomenon : Y. Choquet-Bruhat and N. Noutchegueme in [5] studied the Yang-Mills-Vlasov system using the characteristic method and obtained a local in time existence result. They also studied in [6] the Yang-Mills-Vlasov system only for the zero mass particles case and obtained a global existence theorem in Minkowski space-time for small initial data. N. Noutchegueme and P. Noudjeu in [15] proved a local in time existence theorem of solutions of the Cauchy problem for the Yang-Mills system in temporal gauge with current generated by a distribution function that satisfies a Vlasov equation. R. D. Ayissi and al in [1] obtained the viscosity solutions for the one-Body Liouville equation in Yang-Mills charged Bianchi models with non-zero mass.

We prove here a local in time existence theorem of regular solution for the Boltzmann equation in the presence of a given Yang-Mills field in Minkowski space-time. To our knowledge, there is not such result, even for mild solution, in literature. In this case, unlike in the Abelian's one, the unknow of the 
Boltzmann equation, depends over and above on the position $\left(x^{\alpha}\right)=\left(x^{0}, x^{i}\right)$ and the 4-momentum of particles denoted by $p=\left(p^{\alpha}\right)=\left(p^{0}, p^{i}\right), i=1,2,3$; but also on the non-Abelian charge of particles denoted by $q=\left(q^{a}\right), a=1,2, \ldots, N$; where $N$ is the dimension of the Lie algebra $\mathcal{G}$ of a Lie group $G$. We follow the method used in [2, 11].

The paper is organized as follows : In section 2, we recall some preliminary results and present the equation in the corresponding space-time. Section 3 is devoted to the functional framework and energy estimates. Whereas in section 4 we state and prove the existence and uniqueness theorem.

\section{Preliminary Results and Equations}

- In all what follows, unless otherwise specified, Greek indices range from 0 to 3 and Latin ones from 1 to 3 . We use the Einstein summation convention i.e., $A_{\alpha} B^{\alpha}=\sum_{\alpha} A_{\alpha} B^{\alpha}$.

- We consider the collision evolution of a kind of fast moving massive and charged particles in the Minkowski space-time $\left(\mathbb{R}^{4}, g\right)$, where the metric $g$ is of signature $(-,+,+,+)$. Let $\left(x^{\alpha}\right)=\left(x^{0}, x^{i}\right)$, the global canonical coordinates system on $\mathbb{R}^{4}$, where $x^{0}=t$ is the time coordinate and $x^{i}$ are the spatial coordinates.

- Denote by $A$, a Yang-Mills potential represented by a 1 -form on $\mathbb{R}^{4}$ which takes its values in an $N$-dimensional real Lie algebra $\mathcal{G}$ of a Lie group $G$, endowed with an Ad-invariant scalar product denoted by a dot ".", which enjoys the following property :

$$
f .[k, l]=[f, k] . l, \quad \forall f, k, l \in \mathcal{G},
$$

where [,] stands for the Lie brackets of the Lie algebra $\mathcal{G}$. We consider that $\mathcal{G}$ is a vector space on $\mathbb{R}$ whose dimension is $N \geq 2$ and $\left(\varepsilon_{I}\right)$ an orthonormal basis of $\mathcal{G}$, then the Yang-Mills potential is locally defined as follows :

$$
A=A_{\mu} d x^{\mu}, \text { with } A_{\mu}=A_{\mu}^{I} \varepsilon_{I}, I=1,2, \ldots, N .
$$

- The Yang-Mills field is the curvature of the Yang-Mills potential. It is represented by a $\mathcal{G}$-valued antisymmetric 2-form $F=\left(F_{\lambda \mu}\right)$, linked to the potential $A$ by :

$$
F_{\lambda \mu}^{I}=\nabla_{\lambda} A_{\mu}^{I}-\nabla_{\mu} A_{\lambda}^{I}+\left[A_{\lambda}, A_{\mu}\right]^{I},
$$

with

$$
\left[A_{\lambda}, A_{\mu}\right]^{I}=C_{b c}^{I} A_{\lambda}^{b} A_{\mu}^{c},
$$

where $C_{b c}^{I}$ are the structure constants of $\mathcal{G}$. We require that $A$ verifies the temporal gauge condition, which means that $A_{0}=0$.

- We also suppose that the non-Abelian charge $q$ of the Yang-Mills particles is a $C^{\infty}$ class function from $\mathbb{R}^{4}$ to $\mathcal{G}$ whose given norm is $e>0$. To clarify this idea, $q$ takes its values in a orbit of $\mathcal{G}$, which is a sphere $\vartheta$ whose equation is :

$$
\vartheta: q \cdot q=|q|^{2}=e^{2}
$$

where, $|$.$| stands for the norm deduced from the scalar product.$ 
- The trajectories of particles with momentum $p=\left(p^{\alpha}\right)=\left(p^{0}, \bar{p}\right)$ and charge $q=\left(q^{I}\right)$ in a Yang-Mills field $F$, are no longer geodesics of space-time $\left(\mathbb{R}^{4}, g\right)$, but verify the following differential system :

$$
\frac{d x^{\alpha}}{d s}=p^{\alpha}, \frac{d p^{\alpha}}{d s}=P^{\alpha}, \frac{d q^{I}}{d s}=Q^{I}
$$

where :

$$
P^{\alpha}=-\Gamma_{\lambda \mu}^{\alpha} p^{\lambda} p^{\mu}+p^{\beta} q \cdot F_{\beta}^{\alpha}, Q^{I}=-C_{b c}^{I} p^{\alpha} A_{\alpha}^{b} q^{c},
$$

and $\Gamma_{\lambda \mu}^{\alpha}$ the Christoffel symbols of the Levi-Civita connection associated to $g$. This system expresses the fact that, tangent vector $Y$ to the trajectory of a particle in $\mathbb{P}=T\left(\mathbb{R}^{4}\right) \times \mathcal{G}$ is :

$$
Y=\left(p^{\alpha}, P^{\alpha}, Q^{I}\right)=\left(p^{\alpha},-\Gamma_{\lambda \mu}^{\alpha} p^{\lambda} p^{\mu}+p^{\beta} q \cdot F_{\beta}^{\alpha},-C_{b c}^{I} p^{\alpha} A_{\alpha}^{b} q^{c}\right)
$$

- We consider that particles have the same rest mass $m>0$ normalized to the unity i.e., $m=1$ and a charge $q$ of given size $e$. Then their phase space, that is the domain described by their trajectories is a subset $\mathbb{P}_{1, e}$ of $\mathbb{P}$ whose equation is :

$$
\mathbb{P}_{1, e}=\left\{g_{\alpha \beta} p^{\alpha} p^{\beta}=-1,|q|^{2}=e^{2}\right\} .
$$

Or, using the expression of $g$ and (5), we have :

$$
p^{0}=\left[1+\left(p^{1}\right)^{2}+\left(p^{2}\right)^{2}+\left(p^{3}\right)^{2}\right]^{\frac{1}{2}} \text { and } q^{N}=\left[e^{2}-\sum_{I=1}^{N-1}\left(q^{I}\right)^{2}\right]^{\frac{1}{2}}
$$

where, the choice of $p^{0}>0$ symbolizes the fact that, naturally, the particles eject towards the future. Hence, the local coordinates in $\mathbb{P}_{1, e}=T\left(\mathbb{R}^{4}\right) \times \mathcal{G}$ are $: x^{0}=t, x^{i}, p^{i}, q^{I}$ with, $I=1,2, \ldots, N-1$.

- We denote by $f$ the unknown distribution function which measures the probability density of the presence of particles in a given domain. $f$ is a function defined on $\mathbb{P}_{1, e}$ to $\mathbb{R}$ and will be subject to the Boltzmann equation. Using the fact that we are studying a homogeneous phenomenon, we obtain that the distribution function of Yang-Mills particles is definitely a function of independent variables $\left(t, p^{i}, q^{I}\right)=(t, \bar{p}, \tilde{q})$. Then, $f=f(t, \bar{p}, \tilde{q}), t \in \mathbb{R}, \bar{p} \in \mathbb{R}^{3}, \tilde{q} \in \mathbb{R}^{N-1}$.

- The relativistic Boltzmann equation in $f$ for the Yang-Mills charged particles in Minkowski spacetime can be written :

$$
\frac{\partial f}{\partial t}+\left(-q \cdot F^{i 0}-\frac{p^{j} q \cdot F_{i j}}{p^{0}}\right) \frac{\partial f}{\partial p^{i}}-\frac{p^{i}}{p^{0}} C_{b c}^{I} A_{i}^{b} q^{c} \frac{\partial f}{\partial q^{I}}=\frac{\mathcal{L}(f, f)}{p^{0}} .
$$

The left hand side multiply by $p^{0}$ is in fact the Lie derivative of $f$ with respect to the vectors field $Y$ defined by (8). The right hand side is the collision operator over $p^{0}$, we now introduce :

- According to Lichnerowicz and Chernikov, we consider a scheme in which, at a given position $\left(t, x^{i}\right)$, only two particles collide, without destroying each other, the collision affecting only the momentum and the charge of each particle, which change after the shock; only the sum of the two momenta and the sum of the two charges being preserved. If $\left(p, p_{*}\right)$ (resp. $\left(q, q_{*}\right)$ ) stand for the two momenta (resp. tow charges) before the shock, and $\left(p^{\prime}, p_{*}^{\prime}\right)$ (resp. $\left.\left(q^{\prime}, q_{*}^{\prime}\right)\right)$ stand for the two momenta (resp. tow charges) after the shock, then we have :

$$
\begin{aligned}
& p+p_{*}=p^{\prime}+p_{*}^{\prime} \\
& q+q_{*}=q^{\prime}+q_{*}^{\prime}
\end{aligned}
$$


The collisions operator $\mathcal{L}$ is defined, using two functions $f$ and $g$ on $\mathbb{P}_{1, e}$ and the above notations, by :

$$
\mathcal{L}(f, g)(t, \bar{p}, \tilde{q})=\mathcal{L}^{+}(t, \bar{p}, \tilde{q})-\mathcal{L}^{-}(t, \bar{p}, \tilde{q})
$$

where

$$
\begin{aligned}
& \mathcal{L}^{+}(f, g)=\int_{\mathbb{R}^{3} \times \vartheta} \frac{1}{p_{*}^{0}} d \overline{p_{*}} w_{\tilde{q}_{*}} \int_{S^{2} \times S^{N-2}} f\left(\overline{p^{\prime}}, \tilde{q}^{\prime}\right) g\left(\overline{p_{*}^{\prime}}, \tilde{q_{*}^{\prime}}\right) \sigma d w d \theta \\
& \mathcal{L}^{-}(f, g)=\int_{\mathbb{R}^{3} \times \vartheta} \frac{1}{p_{*}^{0}} d \overline{p_{*}} w_{\tilde{q_{*}}} \int_{S^{2} \times S^{N-2}} f(\bar{p}, \tilde{q}) g\left(\overline{p_{*}}, \tilde{q}_{*}\right) \sigma d w d \theta
\end{aligned}
$$

with :

- $S^{2}$ the unit sphere of $\mathbb{R}^{3}$, whose element is denoted $d w$

- $S^{N-2}$ the unit sphere of $\mathbb{R}^{N-1}$, whose element is denoted $\theta$ and $d \theta$ his volume element

- $\sigma=\sigma\left(t, \bar{p}, \tilde{q}, \overline{p_{*}}, \tilde{q_{*}}, \overline{p^{\prime}}, \tilde{q^{\prime}}, \overline{p_{*}^{\prime}}, \widetilde{q_{*}^{\prime}}\right)$ is a positive regular function called the collision kernel or the crosssection of the collisions, on which we require that

$$
\left(H_{1}\right):\left\{\begin{array}{l}
\exists C_{0}>0,0 \leq \sigma \leq C_{0} \\
(1+|\bar{p}|)^{l}\left\|\partial_{(\bar{p}, \tilde{q})} \sigma\right\|_{L^{1}(\Omega \times \mathfrak{S})} \in L^{\infty}(\Omega), 0 \leq|\beta| \leq m+3,0 \leq l \leq m+3 \\
(1+|\bar{p}|)^{|\beta|-1} \partial_{(\bar{p}, \tilde{q})}^{\beta} \sigma \in L^{\infty}(\Omega \times \Omega \times \mathfrak{S}), 1 \leq|\beta| \leq m+3
\end{array}\right.
$$

where, $\beta \in \mathbb{N}^{m+3}, \Omega=\mathbb{R}^{3} \times \mathbb{R}^{N-1}$ and $\mathfrak{S}=S^{2} \times S^{N-2} . \sigma$ measures interactions effects between particles and determines their nature.

- The conservation law of momenta (12) splits into :

$$
\begin{aligned}
& p^{0}+p_{*}^{0}=p^{\prime 0}+p_{*}^{\prime 0} \\
& \bar{p}+\overline{p_{*}}=\overline{p^{\prime}}+\overline{p_{*}^{\prime}}
\end{aligned}
$$

(15) express the conservation of the quantity $\tilde{e}=\sqrt{1+|\bar{p}|^{2}}+\sqrt{1+\left|\overline{p_{*}}\right|^{2}}$, called the elementary energy of the unit rest mass of particles, we parameterize (16) by setting, following N. Noutchegueme and al in [14], $\bar{p}^{\prime}=\bar{p}+d\left(\bar{p}, \overline{p_{*}}, \omega\right) w$ and $\overline{p_{*}^{\prime}}=\overline{p_{*}}-d\left(\bar{p}, \overline{p_{*}}, \omega\right) w$ with $w \in S^{2}$ in which, $d$ is a regular function given by :

$$
d\left(\bar{p}, \overline{p_{*}}, \omega\right)=\frac{2 \tilde{e} p^{0} p_{*}^{0}\left[\omega \cdot\left(\hat{\bar{p}}_{*}-\hat{\bar{p}}\right)\right]}{\tilde{e}^{2}-\left[\omega \cdot\left(\bar{p}+\bar{p}_{*}\right)\right]^{2}},
$$

where, $\hat{\bar{p}}=\frac{\bar{p}}{p^{0}}, \hat{\bar{p}}_{*}=\frac{\bar{p}_{*}}{p_{*}^{0}}$. The scalar product in (17) is the usual one in $\mathbb{R}^{3}$.

The Jacobian of the transformation $\left(\bar{p}, \overline{p_{*}}\right) \longrightarrow\left(\overline{p^{\prime}}, \overline{p_{*}^{\prime}}\right)$ is given by :

$$
\frac{\partial\left(\overline{p^{\prime}}, \overline{p_{*}^{\prime}}\right)}{\partial\left(\bar{p}, \bar{p}_{*}\right)}=-\frac{p^{\prime 0} p_{*}^{\prime}{ }^{0}}{p^{0} p_{*}^{0}}
$$

The conservation law of charges (13) splits into :

$$
\begin{aligned}
& q^{N}+q_{*}^{N}=q^{\prime N}+q_{*}^{\prime N} \\
& \tilde{q}+\tilde{q}_{*}=\tilde{q}^{\prime}+\tilde{q}_{*}^{\prime} .
\end{aligned}
$$

(19) expresses the conservation of the quantity $\hat{\mathbf{c}}=\left[e^{2}-\sum_{I=1}^{N-1}\left(q^{I}\right)^{2}\right]^{\frac{1}{2}}+\left[e^{2}-\sum_{I=1}^{N-1}\left(q_{*}^{I}\right)^{2}\right]^{\frac{1}{2}}$. 
We can also parameterized (20) by setting, following the sketch given in [14] for the parametrization of the post-collisional momentum :

$$
\left\{\begin{array}{l}
\tilde{q^{\prime}}=\tilde{q}+\eta\left(\tilde{q}, \tilde{q_{*}}, \theta_{1}\right) \theta_{1} \\
\tilde{q_{*}^{\prime}}=\tilde{q_{*}}-\eta\left(\tilde{q}, \tilde{q_{*}}, \theta_{1}\right) \theta_{1}
\end{array}, \quad \theta_{1} \in S^{N-2} .\right.
$$

By raising each member of (19) to square and invoking (21), in the sequel, we get after reduction :

$$
\eta^{2}+\eta\left(\tilde{q}-\tilde{q}_{*} \cdot \theta_{1}\right)+q^{N} q_{*}^{N}-\sqrt{B}=0
$$

$$
\begin{aligned}
& \text { where, } B=\eta^{4}+2 \eta^{3}\left(\tilde{q}-\tilde{q}_{*} \cdot \theta_{1}\right)+\eta^{2}\left[-2 e^{2}+\left(\tilde{q}_{*}\right)^{2}+(\tilde{q})^{2}-4\left(\tilde{q} \cdot \theta_{1}\right)\left(\tilde{q}_{*} \cdot \theta_{1}\right)\right]+ \\
& 2 \eta\left[\left(\tilde{q}_{*}-\tilde{q} \cdot \theta_{1}\right)\left(q_{*}^{N}\right)^{2}+\left\{\left(q^{N}\right)^{2}-\left(q_{*}^{N}\right)^{2}\right\}\left(\tilde{q}_{*} \cdot \theta_{1}\right)\right]+e^{4}-e^{2}\left[\left(\tilde{q}_{*}\right)^{2}+(\tilde{q})^{2}\right]+\left(\tilde{q} \cdot \tilde{q}_{*}\right)^{2} .
\end{aligned}
$$

Multiplying (22) by $\eta^{2}+\eta\left(\tilde{q}-\tilde{q}_{*} \cdot \theta_{1}\right)+q^{N} q_{*}^{N}+\sqrt{B}$, we obtain after computation :

$$
\eta^{2}\left[\left(q^{N}+q_{*}^{N}\right)^{2}+\left(\tilde{q_{*}}+\tilde{q} \cdot \theta_{1}\right)^{2}\right]+2 \eta\left(q^{N}+q_{*}^{N}\right)\left[\left(\tilde{q} \cdot \theta_{1}\right) q_{*}^{N}-\left(\tilde{q_{*}} \cdot \theta_{1}\right) q^{N}\right]=0,
$$

and since $\eta \neq 0$, we finally have :

$$
\eta\left(\tilde{q}, \tilde{q}_{*}, \theta_{1}\right)=\frac{2\left(q^{N}+q_{*}^{N}\right)\left[\left(\tilde{q_{*}} \cdot \theta_{1}\right) q^{N}-\left(\tilde{q} \cdot \theta_{1}\right) q_{*}^{N}\right]}{\left[\left(q^{N}+q_{*}^{N}\right)^{2}+\left\{\left(\tilde{q_{*}}+\tilde{q}\right) \cdot \theta_{1}\right\}^{2}\right]} .
$$

The Jacobian of the transformation $\left(\tilde{q}, \tilde{q_{*}}\right) \longmapsto\left(\tilde{q^{\prime}}, \tilde{q_{*}^{\prime}}\right)$ is given by :

$$
J=\frac{\partial\left(\tilde{q^{\prime}, \tilde{q_{*}^{\prime}}}\right)}{\partial\left(\tilde{q}, \tilde{q_{*}}\right)}=-\frac{q^{\prime} q_{*}^{\prime}}{q^{N} q_{*}^{N}} .
$$

\section{Functional Space and Energy Estimates}

In all what follows, set $\Omega=\mathbb{R}^{3} \times \mathbb{R}^{N-1}$ and $\mathfrak{S}=S^{2} \times S^{N-2}$.

Definition 1. Let $T>0, l \in \mathbb{N}$ and $d \in \mathbb{R}^{+}$.

1. $\mathbb{K}_{d}^{l}(\Omega)=\left\{u: \Omega \longrightarrow \mathbb{R},(1+|\bar{p}|)^{d+|\beta|} \partial_{(\bar{p}, \tilde{q})}^{\beta} u \in L^{2}(\Omega),|\beta| \leq l\right\}$, endowed with the norm $\|u\|_{\mathbb{K}_{d}^{l}(\Omega)}=\max _{0 \leq|\beta| \leq l}\left\|(1+|\bar{p}|)^{d+|\beta|} \partial_{(\bar{p}, \tilde{q})}^{\beta} u\right\|_{L^{2}(\Omega)}$.

2. $\mathbb{K}_{d}^{l}(0, T ; \Omega)=\left\{u \in C([0, T], C(\Omega)),(1+|\bar{p}|)^{d+|\beta|} \partial_{(\bar{p}, \tilde{q})}^{\beta} u(t,.) \in L^{2}(\Omega),|\beta| \leq l\right\}$, endowed with the norm $\|u\|_{\mathbb{K}_{d}^{l}(0, T ; \Omega)}=\sup _{0 \leq t \leq T} \max _{0 \leq|\beta| \leq l}\left\|(1+|\bar{p}|)^{d+|\beta|} \partial_{(\bar{p}, \tilde{q})}^{\beta} u(t, .)\right\|_{L^{2}(\Omega)}$.

3. $\mathbb{E}_{d}^{l}(0, T ; \Omega)=\left\{u \in C([0, T], C(\Omega)),(1+|\bar{p}|)^{d+|\beta|} D_{(\bar{p}, \tilde{q})}^{\beta} u(t,.) \in L^{2}(\Omega),|\beta| \leq l\right\}$, endowed with the norm $\|u\|_{\mathbb{E}_{d}^{l}(0, T ; \Omega)}=\sup _{0 \leq t \leq T} \max _{0 \leq|\beta| \leq l}\left\|(1+|\bar{p}|)^{d+|\beta|} D_{(\bar{p}, \tilde{q})}^{\beta} u(t, .)\right\|_{L^{2}(\Omega)}$.

Where, $D_{(\bar{p}, \tilde{q})}^{\beta}$ denotes the derivative in the sens of distributions. For $\delta>0$, we also define 4. $\mathbb{K}_{d, \delta}^{l}(0, T ; \Omega)=\left\{u \in \mathbb{K}_{d}^{l}(0, T ; \Omega),\|u\|_{\mathbb{K}_{d}^{l}(0, T ; \Omega)} \leq \delta\right\}$

5. $\mathbb{E}_{d, \delta}^{l}(0, T ; \Omega)=\left\{u \in \mathbb{E}_{d}^{l}(0, T ; \Omega),\|u\|_{\mathbb{E}_{d}^{l}(0, T ; \Omega)} \leq \delta\right\}$.

Lemma 1. ([7] 11] Let $T>0, l \in \mathbb{N}$ and $d \in \mathbb{R}$;

i) $\mathbb{E}_{d}^{l}(0, T ; \Omega)$ is a Banach space.

2i) $\mathbb{K}_{d}^{l}(0, T ; \Omega)$ is dense in $\mathbb{E}_{d}^{l}(0, T, \Omega)$ for the norm $\|\cdot\|_{\mathbb{E}_{d}^{l}(0, T, \Omega)}$.

3i) $\mathbb{E}_{d}^{l}(0, T ; \Omega)$ is a separable Hilbert space.

4i) The space $\mathbb{E}_{d, \delta}^{l}(0, T ; \Omega)$ is a complete metric subspace of $\mathbb{E}_{d}^{l}(0, T ; \Omega)$. 
Remark 1. 1) The unknown function $f=f(t, \bar{p}, \tilde{q}) \in \mathbb{R}^{N+2}$ must to be continuously differentiable and has to belong to the space $C_{b}^{1}\left(\mathbb{R}^{N+2}\right)$. We have from Sobolev injection theorem

$$
W_{2}^{l} \hookrightarrow C_{b}^{1}\left(\mathbb{R}^{N+2}\right) \quad \text { if } \quad l>1+\frac{N+2}{2}=\frac{N+4}{2} .
$$

Then, the smallest integer which satisfied this is $l=E\left(\frac{N+4}{2}\right)+1$.

2) If $N=2 m$ or $N=2 m+1$ then, $l=m+3$. From now and on, we will use $l=m+3$.

3) The Sobolev space $\mathbb{E}_{d}^{m+3}(\Omega)$ is a separable Hilbert space $([11] ; p=70)$ since for $t_{0} \in[0, T]$, the norm $\max _{0 \leq|\beta| \leq m+3}\left\|(1+|\bar{p}|)^{d+|\beta|} D_{(\bar{p}, \tilde{q})}^{\beta} u(t, .)\right\|_{L^{2}(\Omega)}$ is equivalent to $\sum_{0 \leq|\beta| \leq m+3}\left\|(1+|\bar{p}|)^{d+|\beta|} D_{(\bar{p}, \tilde{q})}^{\beta} u(t, .)\right\|_{L^{2}(\Omega)}$.

This last norm emanate from the scalar product define for $u, v \in \mathbb{E}_{d}^{m+3}(\Omega)$ by

$(u / v)_{\mathbb{E}_{d}^{m+3}(\Omega)}=\sum_{0 \leq|\beta| \leq m+3}\left((1+|\bar{p}|)^{\frac{1}{2}(d+|\beta|)} D_{(\bar{p}, \tilde{q})}^{\beta} u /(1+|\bar{p}|)^{\frac{1}{2}(d+|\beta|)} D_{(\bar{p}, \tilde{q})}^{\beta} v\right)^{L^{2}(\Omega)}$.

4) For more details on the weighted Sobolev spaces, see [7].

Lemma 2. i) Let $T>0$. The we have:

$$
(\tilde{e})^{2}-\left[w \cdot\left(\bar{p}+\bar{p}_{*}\right)\right]^{2}>2 .
$$

2i) The function $\left(\bar{p}, \bar{p}_{*}, w\right) \mapsto \partial_{\bar{p}}^{\beta} d\left(\bar{p}, \bar{p}_{*}, w\right)$, is bounded for $\beta \in \mathbb{N}^{3}, 1 \leq|\beta| \leq m+3$.

3i) The function $\left(\tilde{q}, \tilde{q}_{*}, \theta\right) \mapsto \partial_{\tilde{q}_{*}}^{\beta} \eta\left(\tilde{q}, \tilde{q}_{*}, \theta\right)$, is bounded for $\beta \in \mathbb{N}^{N-1}, 1 \leq|\beta| \leq m+3$.

Démonstration. i) We have $\left|w \cdot\left(\bar{p}+\overline{p_{*}}\right)\right| \leq|w|\left|\bar{p}+\overline{p_{*}}\right|$, then $(\tilde{e})^{2}-\left[w \cdot\left(\bar{p}+\bar{p}_{*}\right)\right]^{2} \geq(\tilde{e})^{2}-\left(|w|\left|\bar{p}+\overline{p_{*}}\right|\right)^{2}$. Since $|w|^{2}=\left(w_{1}^{2}+w_{2}^{2}+w_{3}^{2}\right)=1$, it then follows that :

$$
(\tilde{e})^{2}-\left[w \cdot\left(\bar{p}+\bar{p}_{*}\right)\right]^{2} \geq(\tilde{e})^{2}-\left|\bar{p}+\overline{p_{*}}\right|^{2}=(\tilde{e})^{2}-\left(|\bar{p}|^{2}+\left|\overline{p_{*}}\right|^{2}+2|\bar{p}|\left|\overline{p_{*}}\right|\right) .
$$

As $(\tilde{e})^{2}=\left(\sqrt{1+|\bar{p}|^{2}}+\sqrt{1+\left|\bar{p}_{*}\right|^{2}}\right)^{2} \geq 2+\left(|\bar{p}|^{2}+\left|\overline{p_{*}}\right|^{2}+2|\bar{p}|\left|\overline{p_{*}}\right|\right)$, using (a), one has (25).

2i) For the second case, let $\beta \in \mathbb{N}^{3}$ such that $1 \leq|\beta| \leq m+3$. If $|\beta|=1$ see [11].

If $|\beta| \geq 2$, we can look $\partial_{\bar{p}}^{\beta} d\left(\bar{p}, \bar{p}_{*}, w\right)$, with $d$ given by (17), as a rational function whose the degree of the denominator is greater or equal to the degree of the numerator and as the order of derivation increases, the degree of the denominator gains more power compared to that of the numerator. So, $\partial_{\bar{p}}^{\beta} d\left(\bar{p}, \bar{p}_{*}, w\right) \longrightarrow 0$ when $|\bar{p}| \longrightarrow+\infty$, hence for $\varepsilon=1$, there exists $\delta>0$ such that $|\bar{p}|>\delta \Rightarrow\left|\partial_{\bar{p}}^{\beta} d\left(\bar{p}, \bar{p}_{*}, w\right)\right|<1$.

In other hand, according to (25), the denominator of the function $\partial_{\bar{p}}^{\beta} d\left(\bar{p}, \bar{p}_{*}, w\right)$ never vanishes, so it's $C^{\infty}$ class function and therefore bounded on the compact $|\bar{p}| \leq \delta$. Hence $2 \mathrm{i}$ ).

$3 \mathrm{i}$ ) is similar to $2 \mathrm{i}$ ).

Lemma 3. The following estimates holds :

i) $(1+|\bar{p}|)^{2} \leq 2\left(p^{0}\right)^{2}$, 2i) $(1+|\bar{p}|) \leq \sqrt{2}\left(1+\left|\overline{p^{\prime}}\right|\right)\left(1+\left|\overline{p_{*}^{\prime}}\right|\right)$ and $\left.3 i\right)(1+|\bar{p}|)^{2} \leq 8 p^{0} p_{*}^{0} p^{\prime}{ }^{0} p_{*}{ }^{0}$.

Démonstration. i) $(1+|\bar{p}|)^{2}=\left(1+\sqrt{\sum_{i=1}^{3}\left(p^{i}\right)^{2}}\right) \leq 2\left(1+\sum_{i=1}^{3}\left(p^{i}\right)^{2}\right)=2\left(p^{0}\right)^{2}$.

$2 i)$ It follows from $i)$ that, $(1+|\bar{p}|) \leq \sqrt{2} p^{0} \leq \sqrt{2}\left(p^{0}+p_{*}^{0}\right)=\sqrt{2}\left(p^{\prime} 0+p_{*}^{\prime 0}\right)$. Besides, $p^{\prime 0} \leq \sqrt{2}\left(1+\left|\overline{p^{\prime}}\right|\right)$ and $p_{*}^{\prime 0} \leq \sqrt{2}\left(1+\left|\overline{p_{*}^{\prime}}\right|\right)$, from where $(1+|\bar{p}|) \leq \sqrt{2}\left(1+\left|\overline{p^{\prime}}\right|\right)\left(1+\left|\overline{p_{*}^{\prime}}\right|\right)$.

3i) Thereafter, combining (2i) and (i), we then have :

$(1+|\bar{p}|)^{2} \leq 4(1+|\bar{p}|)\left(1+\left|\overline{p_{*}}\right|\right)\left(1+\left|\overline{p^{\prime}}\right|\right)\left(1+\left|\overline{p_{*}^{\prime}}\right|\right) \leq 8 p^{0} p_{*}^{0} p^{\prime}{ }^{0} p_{*}^{\prime} 0$. 
Remark 2. Let $\left(a_{i}\right)_{i=1}^{n} \in \mathbb{R}^{n}$. We have :

$$
\left(\sum_{i=1}^{n} a_{i}\right)^{2} \leq n \sum_{i=1}^{n} a_{i}^{2}
$$

Proposition 1. Let $d \in] \frac{N+4}{2},+\infty\left[\right.$. If $f, g \in \mathbb{K}_{d}^{m+3}(\Omega)$ then,

$$
\left\|(1+|\bar{p}|)^{d} \frac{1}{p^{0}} \mathcal{L}^{+}(f, g)\right\|_{L^{2}(\Omega)} \leq C\|f\|_{\mathbb{K}_{d}^{m+3}(\Omega)}\|g\|_{\mathbb{K}_{d}^{m+3}(\Omega)} .
$$

Démonstration. We have :

$$
\begin{aligned}
\left\|(1+|\bar{p}|)^{d} \frac{1}{p^{0}} \mathcal{L}^{+}(f, g)\right\|_{L^{2}(\Omega)}^{2} & =\int_{\Omega}\left[\frac{(1+|\bar{p}|)^{d}}{p^{0}} \int_{\Omega} \frac{1}{p_{*}^{0}} d \bar{p}_{*} w_{\tilde{q}_{*}} \int_{S^{2} \times S^{N-2}} f\left(\overline{p^{\prime}}, \tilde{q}^{\prime}\right) g\left(\overline{p_{*}^{\prime}}, \tilde{q}_{*}^{\prime}\right) \sigma d w d \theta\right]^{2} d \bar{p} w_{\tilde{q}} \\
& =\int_{\Omega} \frac{(1+|\bar{p}|)^{2 d}}{\left(p^{0}\right)^{2}}\left[\int_{\Omega \times \mathfrak{S}} \frac{f\left(\overline{p^{\prime}}, \tilde{q^{\prime}}\right) g\left(\overline{p_{*}^{\prime}}, \tilde{q}_{*}^{\prime}\right)}{p_{*}^{0}} \sigma^{\frac{1}{2}} \sigma^{\frac{1}{2}} d w d \theta d \bar{p}_{*} w_{\tilde{q}_{*}}\right]^{2} d \bar{p} w_{\tilde{q}} .
\end{aligned}
$$

We apply the Cauchy-Schwartz inequality to the integral over $\Omega \times \mathfrak{S}\left(\mathfrak{S}=S^{2} \times S^{N-2}\right)$ to obtain :

$$
\begin{gathered}
\left\|(1+|\bar{p}|)^{d} \frac{1}{p^{0}} \mathcal{L}^{+}(f, g)\right\|_{L^{2}(\Omega)}^{2} \leq \\
C \int_{\mathfrak{S}} d w d \theta \int_{\Omega \times \Omega} \frac{(1+|\bar{p}|)^{2 d}}{p^{0} p_{*}^{0}}\left|f\left(\overline{p^{\prime}}, \tilde{q^{\prime}}\right)\right|^{2}\left|g\left(\overline{p_{*}^{\prime}}, \tilde{q_{*}^{\prime}}\right)\right|^{2} \frac{d \bar{p} d \bar{p}_{*}}{p^{0} p_{*}^{0}} w_{\tilde{q}} w_{\tilde{q}_{*}},
\end{gathered}
$$

since $\|\sigma\|_{L^{1}(\Omega \times \mathfrak{S})} \in L^{\infty}(\Omega)$ and $\sigma$ is bounded. According to (18) and (24), we also know that :

$$
\frac{d \bar{p} d \bar{p}_{*}}{p^{0} p_{*}^{0}}=-\frac{d \bar{p}^{\prime} d \bar{p}_{*}^{\prime}}{p^{\prime} p_{*}^{\prime 0}} \text { and } w_{\tilde{q}} w_{\tilde{q}_{*}}=w_{\tilde{q}^{\prime}} w_{\tilde{q}^{\prime}} .
$$

With $\frac{1}{p^{0} p_{*}^{0}}<1$, (27) gives using 2i) and 3i) in Lemma 3 :

$$
\begin{gathered}
\left\|(1+|\bar{p}|)^{d} \frac{1}{p^{0}} \mathcal{L}^{+}(f, g)\right\|_{L^{2}(\Omega)}^{2} \leq \\
C \int_{\Omega}\left(1+\left|\overline{p^{\prime}}\right|\right)^{2 d}\left|f\left(\bar{p}, \tilde{q^{\prime}}\right)\right|^{2} d \bar{p}^{\prime} w_{\tilde{q}^{\prime}} \times \int_{\Omega}\left(1+\left|\overline{p^{\prime}}{ }_{*}\right|\right)^{2 d}\left|g\left(\overline{p^{\prime}}{ }_{*}, \tilde{q}_{*}^{\prime}\right)\right|^{2} d \overline{p_{*}^{\prime}}{ }_{*} w_{\tilde{q}_{*}^{\prime}} .
\end{gathered}
$$

Then, $\left\|(1+|\bar{p}|)^{d} \frac{1}{p^{0}} \mathcal{L}^{+}(f, g)\right\|_{L^{2}(\Omega)} \leq C\|f\|_{\mathbb{K}_{d}^{m+3}(\Omega)}\|g\|_{\mathbb{K}_{d}^{m+3}(\Omega)}$.

Proposition 2. Let $d \in] \frac{N+4}{2},+\infty\left[\right.$ and $\beta \in \mathbb{N}^{N+2},|\beta|=1$. If $f, g \in \mathbb{K}_{d}^{m+3}(\Omega)$ then :

$$
\left\|(1+|\bar{p}|)^{d+1} \partial_{(\bar{p}, \tilde{q})}^{\beta}\left[\frac{1}{p^{0}} \mathcal{L}^{+}(f, g)\right]\right\|_{L^{2}(\Omega)} \leq C\|f\|_{\mathbb{K}_{d}^{m+3}(\Omega)}\|g\|_{\mathbb{K}_{d}^{m+3}(\Omega)} .
$$

The proof of this proposition will be given by lemmas 4 and 5 ,

Lemma 4. Let $d \in] \frac{N+4}{2},+\infty\left[\right.$ and $i_{1}=1,2,3$. If $f, g \in \mathbb{K}_{d}^{m+3}(\Omega)$, then :

$$
\left\|(1+|\bar{p}|)^{d+1} \frac{\partial}{\partial p^{i_{1}}}\left[\frac{1}{p^{0}} \mathcal{L}^{+}(f, g)\right]\right\|_{L^{2}(\Omega)} \leq C\|f\|_{\mathbb{K}_{d}^{m+3}(\Omega)}\|g\|_{\mathbb{K}_{d}^{m+3}(\Omega)} .
$$


Démonstration. Since $(1+|\bar{p}|) \leq \sqrt{2} p^{0}$ and $\left|p^{i}\right| \leq C p^{0}$, after computation, we have :

$$
\left\|(1+|\bar{p}|)^{d+1} \frac{\partial}{\partial p^{i_{1}}}\left[\frac{1}{p^{0}} \mathcal{L}^{+}(f, g)\right]\right\|_{L^{2}(\Omega)} \leq A_{0}+C B_{0},
$$

where $A_{0}=\left\|\frac{\left(1+|\bar{p}|^{d+1}\right.}{p^{0}} \frac{\partial}{\partial p^{i_{1}}}\left[\mathcal{L}^{+}(f, g)\right]\right\|_{L^{2}(\Omega)}^{2}$ and $B_{0}=\left\|(1+|\bar{p}|)^{d} \frac{1}{p^{0}} \mathcal{L}^{+}(f, g)\right\|_{L^{2}(\Omega)}^{2}$.

The term $B_{0}$ in (29) is estimated in the proposition 11, In the sequel, for the term $A_{0}$, the lemma 2 gives that $\left|\partial_{p^{i_{1}}} \bar{p}^{\prime}\right|$ and $\left|\partial_{p^{i_{1}}} \bar{p}_{*}^{\prime}\right|$ are bounded and using (26), it comes that :

$$
\begin{aligned}
A_{0} & =\int_{\Omega}\left[\frac{(1+|\bar{p}|)^{d+1}}{p^{0}} \int_{\Omega} \frac{1}{p_{*}^{0}} d \bar{p}_{*} w_{\tilde{q}_{*}} \int_{\mathfrak{S}} \frac{\partial}{\partial p^{i_{1}}}\left\{f\left(\overline{p^{\prime}}, \tilde{q^{\prime}}\right) g\left(\overline{p_{*}^{\prime}}, \tilde{q}_{*}^{\prime}\right) \sigma\right\} d w d \theta\right]^{2} d \bar{p} w_{\tilde{q}} \\
& \leq C\left(A_{1}+B_{1}+C_{1}\right),
\end{aligned}
$$

where $\left\{\begin{array}{l}A_{1}=\int_{\Omega} d \bar{p} w_{\tilde{q}} \frac{(1+|\bar{p}|)^{2 d+2}}{\left(p^{0}\right)^{2}}\left[\int_{\Omega \times \mathfrak{S}} \frac{\left|g \sigma \partial_{p^{\prime} i_{1}} f\right|}{p_{*}^{0}} d \overline{p_{*}} d \theta w_{\tilde{q_{*}}} d w\right]^{2} \\ B_{1}=\int_{\Omega} d \bar{p} w_{\tilde{q}} \frac{\left(1+\left.|\bar{p}|\right|^{2 d+2}\right.}{\left(p^{0}\right)^{2}}\left[\int_{\Omega \times \mathfrak{S}} \frac{\left|f \sigma \partial_{p_{*} i_{1} g} g\right|}{p_{*}^{0}} d \overline{p_{*}} d \theta w_{\tilde{q_{*}}} d w\right]^{2} \\ C_{1}=\int_{\Omega} d \bar{p} w_{\tilde{q}} \frac{(1+|\bar{p}|)^{2 d+2}}{\left(p^{0}\right)^{2}}\left[\int_{\Omega \times \mathfrak{S}} \frac{\mid f g \partial_{p_{1} i_{1} \sigma \mid}}{p_{*}^{0}} d \overline{p_{*}} d \theta w_{\tilde{q_{*}}} d w\right]^{2}\end{array}\right.$

For the term $A_{1}$ in (30), using Cauchy-Schwartz's inequality and the assumptions $\left(H_{1}\right)$, we obtain :

$$
\begin{aligned}
A_{1} & \leq \int_{\Omega} d \bar{p} w_{\tilde{q}} \frac{(1+|\bar{p}|)^{2 d+2}}{\left(p^{0}\right)^{2}}\left(\int_{2 \times \mathfrak{S}} \frac{\left|\partial_{p^{\prime i_{1}}} f\right|^{2}|g|^{2} \sigma}{\left(p_{*}^{0}\right)^{2}} d \overline{p_{*}} d \theta w_{\tilde{q}_{*}} d w\right)\left(\int_{\Omega_{\times \mathfrak{S}}} \sigma d \overline{p_{*}} d \theta w_{\tilde{q}_{*}} d w\right) \\
& \leq C \int_{\Omega} \frac{(1+|\bar{p}|)^{2 d+2}}{\left(p^{0}\right)^{2}}\left(\int_{\Omega_{\times \mathfrak{S}}} \frac{\left|\partial_{p^{\prime i_{1}}} f\right|^{2}|g|^{2} \sigma}{\left(p_{*}^{0}\right)^{2}} d \overline{p_{*}} d \theta w_{\tilde{q_{*}}} d w\right) d \bar{p} w_{\tilde{q}} .
\end{aligned}
$$

Considering (28) and the lemma 3 , yields to

$$
\begin{aligned}
A_{1} & \leq C \int_{\mathfrak{S}} d w d \theta \int_{\Omega \times \Omega} \frac{(1+|\bar{p}|)^{2 d+2}}{p^{0} p_{*}^{0}}\left|\partial_{p^{\prime i_{1}}} f\right|^{2}|g|^{2} \frac{d \bar{p} d \overline{p_{*}}}{p^{0} p_{*}^{0}} w_{\tilde{q}} w_{\tilde{q_{*}}} \\
& \leq C \int_{\Omega \times \Omega} \frac{(1+|\bar{p}|)^{2}(1+|\bar{p}|)^{2 d}}{p^{0} p_{*}^{0}}\left|\partial_{p^{\prime_{i}}} f\right|^{2}|g|^{2} \frac{d \bar{p} d \overline{p_{*}}}{p^{0} p_{*}^{0}} w_{\tilde{q}} w_{\tilde{q_{*}}} \\
& \leq C \int_{\Omega \times \Omega} \frac{(1+|\bar{p}|)^{2}\left(1+\left|\overline{p^{\prime}}\right|\right)^{2 d}\left(1+\left|\overline{p_{*}^{\prime}}\right|\right)^{2 d}}{p^{0} p_{*}^{0}}\left|\partial_{p^{\prime i_{1}}} f\right|^{2}|g|^{2} \frac{d \overline{p^{\prime}} d \overline{p_{*}^{\prime}}}{p^{\prime} p_{p_{*}^{\prime}}^{\prime}} w_{\tilde{q}^{\prime}} w_{\tilde{q}_{*}^{\prime}} \\
& \leq C \int_{\Omega}\left(1+\left|\overline{p^{\prime}}\right|\right)^{2 d+2}\left|\partial_{p^{\prime i_{1}}} f\right|^{2} d \overline{p^{\prime}} w_{q^{\prime}} \int_{\Omega}\left(1+\left|\overline{p_{*}^{\prime}}\right|\right)^{2 d}|g|^{2} d \overline{p_{*}^{\prime}} w_{\tilde{q}_{*}^{\prime}}
\end{aligned}
$$

Which means that :

$$
A_{1} \leq C\|f\|_{\mathbb{K}_{d}^{m+3}(\Omega)}^{2}\|g\|_{\mathbb{K}_{d}^{m+3}(\Omega)}^{2} .
$$

For the term $B_{1}$ in (30), interchanging the role play by $f$ and $g$ in $A_{1}$, we obtain an inequality similar to (31) for $B_{1}$. 
Now we have for the last term $C_{1}$ in (30), using Cauchy-Schwartz inequality and $\left(H_{1}\right)$ :

$$
\begin{aligned}
C_{1} & \leq C \int_{\Omega} d \bar{p} w_{\tilde{q}} \frac{(1+|\bar{p}|)^{2 d+2}}{\left(p^{0}\right)^{2}} \int_{\Omega \times \mathfrak{S}} \frac{|f|^{2}|g|^{2} \partial_{p^{i_{1}}} \sigma \mid}{\left(p_{*}^{0}\right)^{2}} d \overline{p_{*}} w_{\tilde{q_{*}}} d w d \theta \int_{\Omega \times \mathfrak{S}}\left|\partial_{p^{\prime i_{1}}} \sigma\right| d \overline{p_{*}} w_{\tilde{q_{*}}} d w d \theta \\
& \leq C \int_{\Omega \times \Omega} \frac{(1+|\bar{p}|)^{2 d+2}}{p^{0} p_{*}^{0}}|f|^{2}|g|^{2} \frac{d \bar{p} d \overline{p_{*}}}{p^{0} p_{*}^{0}} w_{\tilde{q}} w_{\tilde{q_{*}}} .
\end{aligned}
$$

We use (31) in which we replace $\partial_{p^{\prime i_{1}}} f$ by $f$ to obtain :

$$
\begin{aligned}
C_{1} & \leq C \int_{\Omega}\left(1+\left|\overline{p^{\prime}}\right|\right)^{2 d}|f|^{2} d \overline{p^{\prime}} w_{\tilde{q}^{\prime}} \times \int_{\Omega}\left(1+\left|\overline{p_{*}^{\prime}}\right|\right)^{2 d}|g|^{2} d \bar{p}_{*}^{\prime} w_{\tilde{q}_{*}^{\prime}} \\
& \leq C\|f\|_{\mathbb{K}_{d}^{m+3}(\Omega)}^{2}\|g\|_{\mathbb{K}_{d}^{m+3}(\Omega)}^{2} .
\end{aligned}
$$

From (31), (32) and the proposition 1, we have the proof of Lemma 4 .

Lemma 5. let $d \in] \frac{N+4}{2},+\infty\left[\right.$ and $a_{1}=1, \ldots, N-1$. If $f, g \in \mathbb{K}_{d}^{m+3}(\Omega)$, then :

$$
\left\|(1+|\bar{p}|)^{d+1} \frac{\partial}{\partial q^{a_{1}}}\left[\frac{1}{p^{0}} \mathcal{L}^{+}(f, g)\right]\right\|_{L^{2}(\Omega)} \leq C\|f\|_{\mathbb{K}_{d}^{m+3}(\Omega)}^{2}\|g\|_{\mathbb{K}_{d}^{m+3}(\Omega)}^{2} .
$$

Démonstration. Given lemma 2, $\left|\partial_{q^{a_{1}}} \tilde{q}^{\prime}\right|$ and $\left|\partial_{q^{a_{1}}}{\tilde{q_{*}^{\prime}}}_{*}\right|$ are bounded and using (26), we obtain after computation :

$$
\begin{gathered}
\left\|(1+|\bar{p}|)^{d+1} \frac{\partial}{\partial q^{a_{1}}}\left(\frac{1}{p^{0}} \mathcal{L}^{+}(f, g)\right)\right\|_{L^{2}(\Omega)}^{2}= \\
\int_{\Omega} d \bar{p} w_{\tilde{q}}\left[\frac{(1+|\bar{p}|)^{d+1}}{p^{0}} \int_{\Omega} \frac{h^{3}}{p_{*}^{0}} d \overline{p_{*}} w_{\tilde{q_{*}}} \int_{\mathfrak{S}} \frac{\partial}{\partial q^{a_{1}}}\left\{f\left(\overline{p^{\prime}}, \tilde{q^{\prime}}\right) g\left(\overline{p_{*}^{\prime}}, \tilde{q_{*}^{\prime}}\right) \sigma\right\} d w d \theta\right]^{2} \leq A_{2}+B_{2}+C_{2},
\end{gathered}
$$

where $\left\{\begin{array}{l}A_{2}=C \int_{\Omega} d \bar{p} w_{\tilde{q}} \frac{(1+|\bar{p}|)^{2 d+2}}{\left(p^{0}\right)^{2}}\left[\int_{\Omega \times \mathfrak{S}} \frac{\left|\sigma g \partial_{q^{\prime} a_{1}} f\right|}{p_{*}^{0}} d w d \theta d \overline{p_{*}} w_{\tilde{q}_{*}}\right]^{2} \\ B_{2}=C \int_{\Omega} d \bar{p} w_{\tilde{q}} \frac{(1+|\bar{p}|)^{2 d+2}}{\left(p^{0}\right)^{2}}\left[\int_{\Omega \times \mathfrak{S}} \frac{\mid \sigma f \partial_{q_{*}^{\prime} a_{1} g \mid}}{p_{*}^{0}} d w d \theta d \overline{p_{*}} w_{\tilde{q}_{*}}\right]^{2} \\ C_{2}=C \int_{\Omega} d \bar{p} w_{\tilde{q}} \frac{(1+|\bar{p}|)^{2 d+2}}{\left(p^{0}\right)^{2}}\left[\int_{\Omega \times \mathfrak{S}} \frac{\left|f g \partial_{q_{q} a_{1} \sigma \mid} \sigma\right|}{p_{*}^{*}} d w d \theta d \overline{p_{*}} w_{\tilde{q}_{*}}\right]^{2}\end{array}\right.$

For the term $A_{2}$ in (33), using Cauchy-Schwartz inequality and the assumptions $\left(H_{1}\right)$, we have :

$$
\begin{aligned}
A_{2} & \leq \int_{\Omega \times \Omega} \frac{(1+|\bar{p}|)^{2 d+2}}{p^{0} p_{*}^{0}}\left|\partial_{q^{\prime} a_{1}} f\right|^{2}|g|^{2} \frac{d \overline{p_{*}} d \bar{p}}{p_{*}^{o} p^{0}} w_{\tilde{q_{*}}} w_{\tilde{q}} \\
& \leq C \int_{\Omega} \frac{(1+|\bar{p}|)^{2}}{p^{0} p_{*}^{0}}\left(1+\left|\overline{p^{\prime}}\right|\right)^{2 d}\left(1+\left|\overline{p_{*}^{\prime}}\right|\right)^{2 d}\left|\partial_{q^{\prime} a_{1}} f\right|^{2}|g|^{2} \frac{d \overline{p_{*}^{\prime}} d \overline{p^{\prime}}}{p_{*}^{\prime 0} p^{\prime 0}} w_{\tilde{q}^{\prime}} w_{q_{*}^{\prime}} .
\end{aligned}
$$

Which means that :

$$
A_{2} \leq C\|f\|_{\mathbb{K}_{d}^{m+3}(\Omega)}^{2}\|g\|_{\mathbb{K}_{d}^{m+3}(\Omega)}^{2}
$$


For the term $B_{2}$ in (33), interchanging the role play by $f$ and $g$ in $A_{2}$, we obtain an inequality similar to (34) for $B_{2}$.

For the last term $C_{2}$ given by (33), proceeding like in (32), we have :

$$
C_{2} \leq C\|f\|_{\mathbb{K}_{d}^{m+3}(\Omega)}^{2}\|g\|_{\mathbb{K}_{d}^{m+3}(\Omega)}^{2}
$$

(34) and (35) end the proof of lemma 5 .

Lemma 6. There exists a constant $C>0$ such that, $\forall \beta \in \mathbb{N}^{3}$ with $0 \leq|\beta| \leq m+3$, we have :

$$
\left|(1+|\bar{p}|)^{|\beta|} \partial_{\bar{p}}^{\beta}\left(\frac{1}{p^{0}}\right)\right| \leq C \frac{1}{p^{0}} .
$$

Démonstration. For the sake of clarity, we start the proof of this lemma by a conjecture on the module of the multi-index $\beta$, before concluding by a recursion reasoning. Let $\beta \in \mathbb{N}^{3}$ with $0 \leq|\beta| \leq m+3$ and $q(|\beta|)$ be the proposition defined by :

$" \exists C>0$, such that $0 \leq|\beta| \leq m+3$, we have $\left|(1+|\bar{p}|)^{|\beta|} \partial_{\bar{p}}^{\beta}\left(\frac{1}{p^{0}}\right)\right| \leq C \frac{1}{p^{0}} "$

For $|\beta|=0$, the result is obvious, from where $q(0)$ is true.

For $|\beta|=1$, let $i_{1}=1,2,3$, we have $(1+|\bar{p}|) \partial_{p^{i_{1}}}\left(\frac{1}{p^{0}}\right)=-(1+|\bar{p}|) \frac{p^{i_{1}}}{\left(p^{0}\right)^{3}}$. Considering the fact that : $(1+|\bar{p}|) \leq \sqrt{2} p^{0},\left|p^{i_{1}}\right| \leq C p^{0}$ and $\frac{1}{p^{0}}<1$, we obtain :

$$
\left|(1+|\bar{p}|) \partial_{p^{i_{1}}}\left(\frac{1}{p^{0}}\right)\right| \leq C \frac{1}{p^{0}} \text {, from where } q(1) \text { is true. }
$$

For $|\beta|=2$, let $i_{1}, i_{2}=1,2,3$, we have $: \partial_{p^{i_{2}}}\left[\partial_{p^{i_{1}}}\left(\frac{1}{p^{0}}\right)\right]=-\left[\frac{\delta_{i_{1} i_{2}}\left(p^{0}\right)^{3}-3 p^{i_{2}} p^{i_{1}} p^{0}}{\left(p^{0}\right)^{6}}\right]$. Since $(1+|\bar{p}|)^{2} \leq$ $2\left(p^{0}\right)^{2}$ and $\left|p^{i}\right| \leq C p^{0}$, we obtain :

$$
\left|(1+|\bar{p}|)^{2} \partial_{p^{i_{2}} p^{i_{1}}}^{2}\left(\frac{1}{p^{0}}\right)\right| \leq C \frac{1}{p^{0}}, \text { from where } q(2) \text { is true. }
$$

For $|\beta|=3$, let $i_{1}, i_{2}, i_{3}=1,2,3$, we have :

$$
\partial_{p^{i_{3}}}\left[\partial_{p^{i_{2}} p^{i_{1}}}^{2}\left(\frac{1}{p^{0}}\right)\right]=\frac{3 \delta_{i_{1} i_{2}} p^{i_{3}}}{\left(p^{0}\right)^{5}}+\frac{3\left(\delta_{i_{1} i_{3}} p^{i_{2}}+\delta_{i_{2} i_{3}} p^{i_{1}}\right)}{\left(p^{0}\right)^{5}}-\frac{3 p^{i_{1}} p^{i_{2}} p^{i_{3}}}{\left(p^{0}\right)^{7}} .
$$

Likewise, we get :

$$
\left|(1+|\bar{p}|)^{3} \partial_{p^{i_{3}} p^{i_{2}} p^{i_{1}}}\left(\frac{1}{p^{0}}\right)\right| \leq C \frac{1}{p^{0}}, \text { from where } q(3) \text { is true. }
$$

We now suppose that $\forall \beta \in \mathbb{N}^{3}$ such that $3 \leq|\beta| \leq m+2$, the proposition $q(|\beta|)$ is true and show that $q(|\beta|+1)$ is also true.

Let $\beta \in \mathbb{N}^{3}$ such that $3 \leq|\beta| \leq m+2$, in view of the foregoing, we can write :

$$
\partial_{\bar{p}}^{\beta}\left(\frac{1}{p^{0}}\right)=\frac{\chi}{\left(p^{0}\right)^{2|\beta|+1}},
$$

where $\chi$ can be regarded as a polynomial in $\bar{p}$ with bounded coefficients of degree strictly inferior to $2|\beta|+1$.

Let $j=1,2,3$. Using (1A), we have the following relation :

$$
\partial_{p^{j}}\left[\partial_{\bar{p}}^{\beta}\left(\frac{1}{p^{0}}\right)\right]=\frac{\left(\partial_{p^{j}} \chi\right)\left(p^{0}\right)^{2|\beta|}-2(|\beta|+1) p^{j}\left(p^{0}\right)^{2|\beta|-2} \chi}{\left(p^{0}\right)^{4|\beta|+1}},
$$


and taking into account $(1+|\bar{p}|)^{|\beta|} \leq(\sqrt{2})^{|\beta|+1}\left(p^{0}\right)^{|\beta|}$ in the recurrence hypothesis, we established that :

$$
|\chi| \leq C\left(p^{0}\right)^{|\beta|}, \quad(3 A) \quad\left|\partial_{p^{j}} \chi\right| \leq C\left(p^{0}\right)^{|\beta|-1} .
$$

By combining (3A), (4A), $(1+|\bar{p}|)^{|\beta|+1} \leq C(T)\left(p^{0}\right)^{|\beta|+1}$ and $\frac{1}{p^{0}}<1$ in (2A), we obtain :

$$
\left|(1+|\bar{p}|)^{|\beta|+1} \partial_{\bar{p}}^{\beta+\beta^{\prime}}\left(\frac{1}{p^{0}}\right)\right| \leq C \frac{1}{p^{0}}, \forall \beta^{\prime} \in \mathbb{N}^{3} \text { such that }\left|\beta^{\prime}\right|=1,
$$

hence $q(|\beta|+1)$ is true. Which completes the proof of Lemma 6 .

Remark 3. $\forall k \in \mathbb{N}^{N+2}$ with $0 \leq|k| \leq m+3$, there is a unique pair of multi index $\left(k_{1}, k_{2}\right) \in \mathbb{N}^{3} \times \mathbb{N}^{N-1}$ such that $k=\left(k_{1}, k_{2}\right)$. We have

$$
\partial_{(\bar{p}, \tilde{q})}^{k}\left(\frac{1}{p^{0}}\right)=\left\{\begin{array}{l}
\partial_{\bar{p}}^{k}\left(\frac{1}{p^{0}}\right) \text { if } k_{2}=0_{\mathbb{N}^{N-1}} \\
0 \text { else }
\end{array}\right.
$$

Proposition 3. let $d \in] \frac{N+4}{2}, \infty\left[; f, g \in \mathbb{K}_{d}^{m+3}(\Omega)\right.$ and $\beta \in \mathbb{N}^{N+2}$ such that :

$2<|\beta| \leq m+2$. If

$$
\left\|(1+|\bar{p}|)^{d+|\beta|} \partial_{(\bar{p}, \tilde{q})}^{\beta}\left(\frac{1}{p^{0}} \mathcal{L}^{+}(f, g)\right)\right\|_{L^{2}(\Omega)} \leq C\|f\|_{\mathbb{K}_{d}^{m+3}(\Omega)}\|g\|_{\mathbb{K}_{d}^{m+3}(\Omega)}
$$

then, $\forall \beta^{\prime} \in \mathbb{N}^{N+2}$ such that $\left|\beta^{\prime}\right|=1$, we have :

$$
\left\|(1+|\bar{p}|)^{d+|\beta|+1} \partial_{(\bar{p}, \tilde{q})}^{\beta+\beta^{\prime}}\left(\frac{1}{p^{0}} \mathcal{L}^{+}(f, g)\right)\right\|_{L^{2}(\Omega)} \leq C\|f\|_{\mathbb{K}_{d}^{m+3}(\Omega)}\|g\|_{\mathbb{K}_{d}^{m+3}(\Omega)}
$$

Démonstration. By setting

$$
\alpha=\beta+\beta^{\prime} \text { we have }|\alpha| \leq|\beta|+\left|\beta^{\prime}\right| \leq m+3,
$$

and using the Leibniz formula, we obtain :

$$
\partial_{(\bar{p}, \tilde{q})}^{\beta+\beta^{\prime}}\left(\frac{1}{p^{0}} \mathcal{L}^{+}(f, g)\right)=\frac{1}{p^{0}} \partial_{(\bar{p}, \tilde{q})}^{\alpha}\left(\mathcal{L}^{+}\right)+\sum_{k \leq \alpha,|k| \geq 1} C_{\alpha}^{k} \partial_{(\bar{p}, \tilde{q})}^{k}\left(\frac{1}{p^{0}}\right) \partial_{(\bar{p}, \tilde{q})}^{\alpha-k}\left(\mathcal{L}^{+}\right)
$$

Which imply

$$
\left\|(1+|\bar{p}|)^{d+|\alpha|} \partial_{(\bar{p}, \tilde{q})}^{\alpha}\left(\frac{1}{p^{0}} \mathcal{L}^{+}(f, g)\right)\right\|_{L^{2}(\Omega)} \leq A_{1}+A_{2},
$$

where :

$$
\left\{\begin{array}{l}
A_{1}=\left\|(1+|\bar{p}|)^{d+|\alpha|} \frac{1}{p^{0}} \partial_{(\bar{p}, \tilde{q})}^{\alpha} \mathcal{L}^{+}\right\|_{L^{2}(\Omega)}^{2} \\
A_{2}=\left\|(1+|\bar{p}|)^{d+|\alpha|} \sum_{k \leq \alpha,|k| \geq 1} C_{\alpha}^{k} \partial_{(\bar{p}, \tilde{q})}^{k}\left(\frac{1}{p^{0}}\right) \partial_{(\bar{p}, \tilde{q})}^{\alpha-k}\left(\mathcal{L}^{+}\right)\right\|_{L^{2}(\Omega)}^{2}
\end{array}\right.
$$

In $A_{2}$ given by (37), perform the following factorization

$$
A_{2}=\left\|(1+|\bar{p}|)^{d+|\alpha|} \sum_{k \leq \alpha,|k| \geq 1} C_{\alpha}^{k}(1+|\bar{p}|)^{|k|} \partial_{(\bar{p}, \tilde{q})}^{k}\left(\frac{1}{p^{0}}\right) \partial_{(\bar{p}, \tilde{q})}^{\alpha-k}\left(\mathcal{L}^{+}\right) \frac{1}{(1+|\bar{p}|)^{|k|}}\right\|_{L^{2}(\Omega)}^{2} .
$$


Using lemma 6, we have :

$$
A_{2} \leq C\|f\|_{\mathbb{K}_{d}^{m+3}(\Omega)}^{2}\|g\|_{\mathbb{K}_{d}^{m+3}(\Omega)}^{2}
$$

since $|\alpha|-|k| \leq m+2$ because $|k| \geq 1$ and $|\alpha| \leq m+3$, we conclude from the recurrence hypothesis.

We then have to study $A_{1}$ given by (37). one has

$$
\partial_{(\bar{p}, \tilde{q})}^{\alpha} \mathcal{L}^{+}(f, g)=\int_{\Omega} \frac{1}{p_{*}^{0}} d \bar{p}_{*} w_{\tilde{q_{*}}} \int_{\mathfrak{S}} \partial_{(\bar{p}, \tilde{q})}^{\alpha}(f g \sigma) d w d \theta
$$

Applying twice successively the Leibniz's formula we obtain :

$$
\partial_{(\bar{p}, \tilde{q})}^{\alpha}(f g \sigma)=\sum_{k \leq \alpha} C_{\alpha}^{k} \partial_{(\bar{p}, \tilde{q})}^{\alpha-k}(\sigma) \sum_{\lambda \leq k} C_{k}^{\lambda} \partial_{(\bar{p}, \tilde{q})}^{\lambda}(f) \partial_{(\bar{p}, \tilde{q})}^{k-\lambda}(g)
$$

Which yields

$$
A_{1}=\int_{\Omega} d \bar{p} w_{\tilde{q}}\left[\frac{(1+|\bar{p}|)^{d+|\alpha|}}{p^{0}} \int_{\Omega} \frac{1}{p_{*}^{0}} d \overline{p_{*}} w_{\tilde{q_{*}}} \int_{\mathfrak{S}} \sum_{k \leq \alpha} C_{\alpha}^{k} \partial_{(\bar{p}, \tilde{q})}^{\alpha-k}(\sigma) \sum_{\lambda \leq k} C_{k}^{\lambda} \partial_{(\bar{p}, \tilde{q})}^{\lambda}(f) \partial_{(\bar{p}, \tilde{q})}^{k-\lambda}(g) d w d \theta\right]^{2} .
$$

By using (26), we get :

$$
A_{1} \leq C \sum_{k \leq \alpha} J_{k}
$$

where : $J_{k}=\int_{\Omega} d \bar{p} w_{\tilde{q}}\left[\frac{(1+|\bar{p}|)^{d+|\alpha|}}{p^{0}} \int_{\Omega} \frac{1}{p_{*}^{0}} d \bar{p}_{*} w_{\tilde{q_{*}}} \int_{\mathfrak{S}} \partial_{(\bar{p}, \tilde{q})}^{\alpha-k}(\sigma) \sum_{\lambda \leq k} C_{k}^{\lambda} \partial_{(\bar{p}, \tilde{q})}^{\lambda}(f) \partial_{(\bar{p}, \tilde{q})}^{k-\lambda}(g) d w d \theta\right]^{2}$.

Let $s_{1} \in \mathbb{N}^{m+3}$, arbitrarily chosen and satisfying $s_{1} \leq \alpha$. For $k=s_{1}$, we have according to (26) :

$$
J_{s_{1}} \leq C \sum_{\lambda \leq s_{1}} I_{\lambda}
$$

where, $I_{\lambda}=\int_{\Omega} d \bar{p} w_{\tilde{q}}\left[\frac{(1+|\bar{p}|)^{d+|\alpha|}}{p^{0}} \int_{\Omega} \frac{1}{p_{*}^{0}} d \bar{p}_{*} w_{\tilde{q_{*}}} \int_{\mathfrak{S}} \partial_{(\bar{p}, \tilde{q})}^{\alpha-s_{1}}(\sigma) \partial_{(\bar{p}, \tilde{q})}^{\lambda}(f) \partial_{(\bar{p}, \tilde{q})}^{s_{1}-\lambda}(g) d w d \theta\right]^{2}$. let $s_{2} \in \mathbb{N}^{N+2}$, arbitrarily chosen and satisfying $s_{2} \leq s_{1}$.

Estimation of $I_{\lambda}$ for $\lambda=s_{2}$

Apply the Cauchy-Schwartz inequality for the integral on $\Omega \times \mathfrak{S}$, we get by considering the assumptions on the collision kernel $\left(H_{1}\right)$ :

$$
I_{s_{2}} \leq C \int_{\Omega} d \bar{p} w_{\tilde{q}} \frac{(1+|\bar{p}|)^{2 d+2|\alpha|}}{\left(p^{0}\right)^{2}} \int_{\Omega \times \mathfrak{S}} \frac{\left|\partial_{(\bar{p}, \tilde{q})}^{s_{2}} f\right|^{2}\left|\partial_{(\bar{p}, \tilde{q})}^{s_{1}-s_{2}} g\right|^{2}}{\left(p_{*}^{0}\right)^{2}} d \bar{p}_{*} w_{\tilde{q_{*}}} d w d \theta
$$

Using the lemma 3 and the inequality $|\alpha| \leq|\beta|+1$ we have :

$$
\begin{aligned}
I_{s_{2}} & \leq C \int_{\Omega \times \Omega} \frac{(1+|\bar{p}|)^{2 d+2|\alpha|}\left|\partial^{s_{2}} f\right|^{2}\left|\partial^{s_{1}-s_{2}} g\right|^{2}}{\left(p_{*}^{0}\right)^{2}\left(p^{0}\right)^{2}} d \overline{p_{*}} w_{\tilde{q}_{*}} d \bar{p} w_{\tilde{q}} \\
& \leq C \int_{\Omega \times \Omega}\left(1+\left|\overline{p^{\prime}}\right|\right)^{2 d+2|\beta|}\left|\partial^{s_{2}} f\right|^{2}\left(1+\left|\overline{p_{*}^{\prime}}\right|\right)^{2 d+2|\beta|}\left|\partial^{s_{1}-s_{2}} g\right|^{2} \frac{(1+|\bar{p}|)^{2}}{p_{*}^{0} p^{0}} \frac{d \bar{p} d \overline{p_{*}}}{p_{*}^{0} p^{0}} w_{\tilde{q}_{*}} w_{\tilde{q}}
\end{aligned}
$$




$$
\begin{aligned}
& \leq C \int_{\Omega \times \Omega}\left(1+\left|\overline{p^{\prime}}\right|\right)^{2 d+2|\beta|}\left|\partial^{s_{2}} f\right|^{2}\left(1+\left|\overline{p_{*}^{\prime}}\right|\right)^{2 d+2|\beta|}\left|\partial^{s_{1}-s_{2}} g\right|^{2} d \overline{p^{\prime}} d \overline{p_{*}^{\prime}} w_{{\tilde{q^{\prime}}}^{\prime}} w_{\tilde{q}^{\prime}} \\
& \leq C \int_{\Omega}\left(1+\left|\overline{p^{\prime}}\right|\right)^{2 d+2|\beta|}\left|\partial^{s_{2}} f\right|^{2} d \overline{p^{\prime}} w_{\tilde{q}^{\prime}} \int_{\Omega}\left(1+\left|\overline{p_{*}^{\prime}}\right|\right)^{2 d+2|\beta|}\left|\partial^{s_{1}-s_{2}} g\right|^{2} d \overline{p_{*}^{\prime}} w_{\tilde{q}^{\prime}} .
\end{aligned}
$$

Which means :

$$
I_{s_{2}} \leq C\|f\|_{\mathbb{K}_{d}^{m+3}(\Omega)}^{2}\|g\|_{\mathbb{K}_{d}^{m+3}(\Omega)}^{2}
$$

As $s_{2}$ is arbitrarily chosen, we have :

$$
J_{s_{1}} \leq C \sum_{\lambda=0}^{s_{1}} I_{\lambda} \leq C\|f\|_{\mathbb{K}_{d}^{m+3}(\Omega)}^{2}\|g\|_{\mathbb{K}_{d}^{m+3}(\Omega)}^{2}
$$

Likewise, as $s_{1}$ is arbitrarily chosen in (41)

$$
A_{1} \leq C \sum_{k \leq \alpha} J_{k} \leq C\|f\|_{\mathbb{K}_{d}^{m+3}(\Omega)}^{2}\|g\|_{\mathbb{K}_{d}^{m+3}(\Omega)}^{2}
$$

(38) and (42) end the proof of proposition 3.

Theorem 1. Let $d \in] \frac{N+4}{2},+\infty\left[\right.$ and $f, g \in \mathbb{K}_{d}^{m+3}(\Omega)$. We have :

i)

$$
\frac{1}{p^{0}} \mathcal{L}^{+}(f, g) \in \mathbb{K}_{d}^{m+3}(\Omega), \quad \frac{1}{p^{0}} \mathcal{L}^{-}(f, g) \in \mathbb{K}_{d}^{m+3}(\Omega) .
$$

ii) There exists $C>0$ such that

$$
\begin{aligned}
& \left\|\frac{1}{p^{0}} \mathcal{L}^{+}(f, g)\right\|_{\mathbb{K}_{d}^{m+3}(\Omega)} \leq C\|f\|_{\mathbb{K}_{d}^{m+3}(\Omega)}\|g\|_{\mathbb{K}_{d}^{m+3}(\Omega)} . \\
& \left\|\frac{1}{p^{0}} \mathcal{L}^{-}(f, g)\right\|_{\mathbb{K}_{d}^{m+3}(\Omega)} \leq C\|f\|_{\mathbb{K}_{d}^{m+3}(\Omega)}\|g\|_{\mathbb{K}_{d}^{m+3}(\Omega)} .
\end{aligned}
$$

Démonstration. We show that, $\forall \beta \in \mathbb{N}^{N+2}$ such that $0 \leq|\beta| \leq m+3$ we have :

$$
\left\|(1+|\bar{p}|)^{d+|\beta|} \partial_{(\bar{p}, \tilde{q})}^{\beta}\left(\frac{1}{p^{0}} \mathcal{L}^{+}(f, g)\right)\right\|_{L^{2}(\Omega)} \leq C\|f\|_{\mathbb{K}_{d}^{m+3}(\Omega)}\|g\|_{\mathbb{K}_{d}^{m+3}(\Omega)} .
$$

For that, we reason by recurrence on the module of the multi index $\beta$. The conclusion is obtained using propositions 1, 2 and 3, For the term in $\mathcal{L}^{-}(f, g)$ we proceed in the same way.

Lemma 7. Let $d \in] \frac{N+4}{2},+\infty\left[\right.$ and $f, g \in \mathbb{E}_{d}^{m+3}(\Omega)$. We have :

l1) $\begin{aligned} & \left\|\frac{1}{p^{0}} \mathcal{L}^{+}(f, f)-\frac{1}{p^{0}} \mathcal{L}^{+}(g, g)\right\|_{\mathbb{K}_{d}^{m+3}(\Omega)} \leq C\left(\|f\|_{\mathbb{K}_{d}^{m+3}(\Omega)}+\|g\|_{\mathbb{K}_{d}^{m+3}(\Omega)}\right)\|f-g\|_{\mathbb{K}_{d}^{m+3}(\Omega)} \\ & \text { l2) }\left\|\frac{1}{p^{0}} \mathcal{L}^{-}(f, f)-\frac{1}{p^{0}} \mathcal{L}^{-}(g, g)\right\|_{\mathbb{K}_{d}^{m+3}(\Omega)} \leq C\left(\|f\|_{\mathbb{K}_{d}^{m+3}(\Omega)}+\|g\|_{\mathbb{K}_{d}^{m+3}(\Omega)}\right)\|f-g\|_{\mathbb{K}_{d}^{m+3}(\Omega)} \text {. }\end{aligned}$

Démonstration. For the proof of this lemma, we use the bilinearity of the collision operator. 
Theorem 2. Let $d \in] \frac{N+4}{2},+\infty\left[\right.$ and $f, g \in \mathbb{E}_{d}^{m+3}(\Omega)$. then, we have :

i)

$$
\frac{1}{p^{0}} \mathcal{L}(f, g) \in \mathbb{E}_{d}^{m+3}(\Omega)
$$

ii) There exists $C>0$ such that

$$
\left\|\frac{1}{p^{0}} \mathcal{L}(f, g)\right\|_{\mathbb{E}_{d}^{m+3}(\Omega)} \leq C\|f\|_{\mathbb{E}_{d}^{m+3}(\Omega)}\|g\|_{\mathbb{E}_{d}^{m+3}(\Omega)} .
$$

iii) $\left\|\frac{1}{p^{0}} \mathcal{L}(f, f)-\frac{1}{p^{0}} \mathcal{L}(g, g)\right\|_{\mathbb{E}_{d}^{m+3}(\Omega)} \leq 2 C\left(\|f\|_{\mathbb{E}_{d}^{m+3}(\Omega)}+\|g\|_{\mathbb{E}_{d}^{m+3}(\Omega)}\right)\|f-g\|_{\mathbb{E}_{d}^{m+3}(\Omega)}$.

Démonstration. a) We use the density of $\mathbb{K}_{d}^{m+3}(\Omega)$ in $\mathbb{E}_{d}^{m+3}(\Omega)$ and therefore, reason for $f$ and $g \in$ $\mathbb{K}_{d}^{m+3}(\Omega)$ to conclude classically that $\frac{1}{p^{0}} \mathcal{L}(f, g) \in \mathbb{E}_{d}^{m+3}(\Omega)$.

Let $f, g \in \mathbb{K}_{d}^{m+3}(\Omega)$ and $\beta \in \mathbb{N}^{m+3}, 0 \leq|\beta| \leq m+3$, we have :

$$
\frac{1}{p^{0}} \mathcal{L}(f, g)=\frac{1}{p^{0}} \mathcal{L}^{+}(f, g)-\frac{1}{p^{0}} \mathcal{L}^{-}(f, g) .
$$

We conclude using the theorem 2 that $\frac{1}{p^{0}} \mathcal{L}(f, g) \in \mathbb{K}_{d}^{m+3}(\Omega)$, and there is $C>0$ such that $\left\|\frac{1}{p^{0}} \mathcal{L}(f, g)\right\|_{\mathbb{K}_{d}^{m+3}(\Omega)} \leq C\|f\|_{\mathbb{K}_{d}^{m+3}(\Omega)}\|g\|_{\mathbb{K}_{d}^{m+3}(\Omega)}$.

b) For iii), we use the bilinearity of collision operator and lemma 7

\section{Existence and Uniqueness Theorem}

We will prove that, the Boltzmann equation which writes :

$$
\frac{\partial f}{\partial t}+P^{i} \frac{\partial f}{\partial p^{i}}+Q^{I} \frac{\partial f}{\partial q^{I}}=\frac{1}{p^{0}} \mathcal{L}(f, f)
$$

with $f(0, \bar{p}, \tilde{q})=f_{0} \in \mathbb{E}_{d, \delta}^{m+3}(\Omega)$, has in $\mathbb{E}_{d, \delta}^{m+3}(\Omega)$ a local unique and bounded solution.

Where $P^{i}=-\left(q \cdot F^{i 0}+\frac{p^{j} q \cdot F_{i j}}{p^{0}}\right)$ and $Q^{I}=-\frac{p^{i}}{p^{0}} C_{b c}^{I} A_{i}^{b} q^{c}$.

We will suppose that the Yang-Mills potential $A$ and the Yang-Mills field $F$ are given in $C_{0}^{\infty}\left(\left[0,+\infty\left[\times \mathbb{R}^{3}\right)\right.\right.$, the space of restrictions to $\left[0,+\infty\left[\times \mathbb{R}^{3}\right.\right.$ of $C^{\infty}$ functions, or $\mathcal{G}$-values tensors, with compact support on $\mathbb{R}^{4}$.

Lemma 8. $i)$ Let $t \in[0, T[$, the application $t \longrightarrow \bar{p}(t)$ is uniformly bounded.

ii) $P^{i}$ and $Q^{I}$ are bounded.

iii) $(1+|\bar{p}|)^{|\beta|-1} \partial_{(\bar{p}, \tilde{q})}^{\beta} P^{i}$ is bounded, for all $\beta \in \mathbb{N}^{N+2}$ with $1 \leq|\beta| \leq m+3$,

iv) $(1+|\bar{p}|)^{|\beta|-1} \partial_{(\bar{p}, \tilde{q})}^{\beta} Q^{I}$ is bounded, for all $\beta \in \mathbb{N}^{N+2}$ with $1 \leq|\beta| \leq m+3$.

Démonstration. Knowing that in the Minkowski space-time, $\Gamma_{\lambda \mu}^{\alpha}=0$, using (6) we have, $\frac{d p^{i}}{d s}=-\left(q \cdot F^{i 0}+\frac{p^{j} q \cdot F_{i j}}{p^{0}}\right)$. Combining (5) $), \frac{1}{p^{0}}$ and the fact that $F \in C_{0}^{\infty}\left(\left[0,+\infty\left[\times \mathbb{R}^{3}\right)\right.\right.$, we get $\left|\dot{p}^{i}(t)\right| \leq C \sum_{j=1}^{3}\left|p^{j}(t)\right|+C^{i}$, where $C^{i}=C(e,|F|)$ and the dot stands for the derivative with respect to $t$.

By setting $C_{1}=\sum_{i=1}^{3} C^{i}=C(e,|F|)$, it follows that :

$$
|\dot{\bar{p}}(t)| \leq C|\bar{p}(t)|+C_{1}
$$


In the sequel, integrating relation (44) over $[0, t], t \in[0, T[$, one obtains

$$
|\bar{p}(t)| \leq|\bar{p}(0)|+C_{1} T+C \int_{0}^{t}|\bar{p}(s)| d s .
$$

Applying the Gronwall lemma, one has $|\bar{p}(t)| \leq\left(|\bar{p}(0)|+C_{1} T\right) e^{C T}, t \in[0, T[$.

ii) is immediate using i) and the fact that the Yang-Mills potential $A$ and the Yang-Mills field $F$ are bounded.

iii) For all $\beta \in \mathbb{N}^{N+2}$ such that $1 \leq|\beta| \leq m+3$, we have :

$$
\partial_{(\bar{p}, \tilde{q})}^{\beta} P^{i}=-\partial_{(\bar{p}, \tilde{q})}^{\beta}\left(q \cdot F^{i 0}\right)-\partial_{(\bar{p}, \tilde{q})}^{\beta}\left(\frac{p^{j} q \cdot F_{i j}}{p^{0}}\right) .
$$

Knowing that the application $t \longrightarrow \bar{p}(t)$ is uniformly bounded on $[0, T[$, immediately

$$
(1+|\bar{p}|)^{|\beta|-1}\left|\partial_{(\bar{p}, \tilde{q})}^{\beta}\left(q \cdot F^{i 0}\right)\right| \leq C(T, e,|F|) .
$$

Furthermore, the Leibniz's formula applied twice successively gives

$$
\begin{aligned}
\partial_{(\bar{p}, \tilde{q})}^{\beta}\left(\frac{p^{j} g^{i i} q \cdot F_{i j}}{p^{0}}\right) & =\sum_{k \leq \beta} C_{\beta}^{k} \partial_{(\bar{p}, \tilde{q})}^{k}\left(\frac{1}{p^{0}}\right) \sum_{\lambda \leq \beta-k} C_{\beta-k}^{\lambda} \partial_{(\bar{p}, \tilde{q})}^{\lambda} p^{j} \partial_{(\bar{p}, \tilde{q})}^{\beta-\lambda}\left(q \cdot F_{i j}\right) \\
& =\sum_{k \leq \beta} C_{\beta}^{k}(1+|\bar{p}|)^{|k|} \partial_{(\bar{p}, \tilde{q})}^{k}\left(\frac{1}{p^{0}}\right) \sum_{\lambda \leq \beta-k} C_{\beta-k}^{\lambda} \partial_{(\bar{p}, \tilde{q})}^{\lambda} p^{j} \partial_{(\bar{p}, \tilde{q})}^{\beta-k-\lambda}\left(q \cdot F_{i j}\right) \times \frac{1}{(1+|\bar{p}|)^{|k|}} .
\end{aligned}
$$

Invoking the lemma 6 and the fact that $\frac{1}{(1+|\bar{p}|)^{|k|}}<1$, we get :

$\left|(1+|\bar{p}|)^{|\beta|-1} \partial_{(\bar{p}, \tilde{q})}^{\beta}\left(\frac{p^{j} g^{i i} q \cdot F_{i j}}{p^{0}}\right)\right| \leq C(1+|\bar{p}|)^{|\beta|-1} \sum_{k \leq \beta} C_{\beta}^{k} \frac{1}{p^{0}} \sum_{\lambda \leq \beta-k} C_{\beta-k}^{\lambda}\left|\partial_{(\bar{p}, \tilde{q})}^{\lambda} p^{j}\right|\left|\partial_{(\bar{p}, \tilde{q})}^{\beta-k-\lambda}\left(q \cdot F_{i j}\right)\right|$,

we conclude that :

$\left|(1+|\bar{p}|)^{|\beta|-1} \partial_{(\bar{p}, \tilde{q})}^{\beta}\left(\frac{p^{j} g^{i i} q \cdot F_{i j}}{p^{0}}\right)\right| \leq C(T, e,|F|)$.

(1B) and (2B) complete the proof of iii). The proof for iv) is similar to iii)

Let $\tilde{f} \in \mathbb{E}_{d, \delta}^{m+3}(\Omega)$. The linearized Boltzmann equation in $f$ is the equation

$$
\frac{\partial f}{\partial t}+P^{i} \frac{\partial f}{\partial p^{i}}+Q^{I} \frac{\partial f}{\partial q^{I}}=\frac{1}{p^{0}} \mathcal{L}(\tilde{f}, \tilde{f})
$$

with $f(0, \bar{p}, \tilde{q})=f_{0}$. Where $P^{i}=-\left(q \cdot F^{i 0}+\frac{p^{j} g^{i i} q \cdot F_{i j}}{p^{0}}\right)$ and $Q^{I}=-\frac{p^{i}}{p^{0}} C_{b c}^{I} A_{i}^{b} q^{c}$.

We will prove that (45) has in $\mathbb{E}_{d}^{m+3}(\Omega)$ a local unique and bounded solution. We use the Faedo-Galerking method by constructing a sequence and we prove that this sequence is bounded in $\mathbb{E}_{d}^{m+3}(\Omega)$.

\section{Construction of the sequence}

Let $\tilde{f}$ be given in $\mathbb{E}_{d}^{m+3}(\Omega)$. Let $\left(v_{k}\right)_{k \in \mathbb{N}^{*}}$ be a hilbertian basis of $\mathbb{E}_{d}^{m+3}(\Omega)$ (since it is a separable Hilbert space). We follow the method used in [2] for the construction of the sequence $\left(f^{M}\right)_{M}$ which will converge to the solution $f$ of (45). We then write

$$
f^{M}=\sum_{k=1}^{M} \lambda_{k} v_{k}, M \in \mathbb{N}^{*}
$$


where the coefficients $\lambda_{k}$ are derivable functions of $t$ and are given as solutions of the following system of linear first order differential equations :

$$
\left(\partial_{t} f^{M} / v_{j}\right)+\left(P^{i} \partial_{p^{i}} f^{M} / v_{j}\right)+\left(Q^{I} \partial_{q^{I}} f^{M} / v_{j}\right)=\left(\frac{1}{p^{0}} \mathcal{L}(\tilde{f}, \tilde{f}) / v_{j}\right),
$$

with the initial data :

$$
\lambda_{j}(0)=\left(f_{0} / v_{j}\right)
$$

where (./.) stands for the scalar product in $\mathbb{E}_{d}^{m+3}$. Thus we obtain, that :

$$
\partial_{t} f^{M}+P^{i} \partial_{p^{i}} f^{M}+Q^{I} \partial_{q^{I}} f^{M}=\frac{1}{p^{0}} \mathcal{L}(\tilde{f}, \tilde{f}) .
$$

So $f^{M}$ is solution of (45), with the initial data :

$$
f^{M}(0)=\sum_{k=1}^{M} \lambda_{k}(0) v_{k}=\sum_{k=1}^{M}\left(f_{0} / v_{k}\right) v_{k}
$$

Proposition 4. Let $d \in] \frac{N+4}{2}, \infty\left[, f^{M} \in \mathbb{E}_{d}^{m+3}(\Omega), \alpha, \beta \in \mathbb{N}^{N+2},|\alpha| \leq|\beta| \leq m+3\right.$, then

$$
\begin{gathered}
\left|\left((1+|\bar{p}|)^{d+|\beta|} \partial_{(\bar{p}, \tilde{q})}^{\beta}\left[P^{i} \partial_{p^{i}} f^{M}\right] /(1+|\bar{p}|)^{d+|\beta|} \partial_{(\bar{p}, \tilde{q})} f^{M}\right)_{L^{2}(\Omega)}\right| \leq \\
C(T, e,|F|)\left(\sum_{|\alpha| \leq|\beta|}\left\|(1+|\bar{p}|)^{d+|\alpha|} \partial_{(\bar{p}, \tilde{q})}^{\alpha} f^{M}\right\|_{L^{2}(\Omega)}\right)\left\|(1+|\bar{p}|)^{d+|\beta|} \partial_{(\bar{p}, \tilde{q})}^{\beta} f^{M}\right\|_{L^{2}(\Omega)}
\end{gathered}
$$

where : $C=C(T, e,|F|)$ and $(/)$ stands for the scalar product in $L^{2}(\Omega)$.

The proof of this proposition is given by Lemmas 9,10 and 11 .

Lemma 9. Let $d \in] \frac{N+4}{2}, \infty\left[\right.$ and $f^{M} \in \mathbb{E}_{d}^{m+3}(\Omega)$, then :

$$
\left|\left((1+|\bar{p}|)^{d}\left[P^{i} \frac{\partial f^{M}}{\partial p^{i}}\right] /(1+|\bar{p}|)^{d} f^{M}\right)_{L^{2}(\Omega)}\right| \leq C(T, e,|F|)\left\|(1+|\bar{p}|)^{d} f^{M}\right\|_{L^{2}(\Omega)}^{2} .
$$

Démonstration. We have $\partial_{p^{i}}\left[(1+|\bar{p}|)^{d} f^{M}\right]=\partial_{p^{i}}\left[(1+|\bar{p}|)^{d}\right] f^{M}+(1+|\bar{p}|)^{d} \partial_{p^{i}} f^{M}$, from where

$$
\left|\left((1+|\bar{p}|)^{d} P^{i}\left[\frac{\partial f^{M}}{\partial p^{i}}\right] /(1+|\bar{p}|)^{d} f^{M}\right)_{L^{2}(\Omega)}\right| \leq\left|B_{1}\right|+\left|B_{2}\right|,
$$

with $\left\{\begin{array}{l}B_{1}=\left(P^{i} \partial_{p^{i}}\left[(1+|\bar{p}|)^{d} f^{M}\right] /(1+|\bar{p}|)^{d} f^{M}\right)_{L^{2}(\Omega)} \\ B_{2}=\left(P^{i} \partial_{p^{i}}\left[(1+|\bar{p}|)^{d}\right] f^{M} /(1+|\bar{p}|)^{d} f^{M}\right)_{L^{2}(\Omega)}\end{array}\right.$

For the term $B_{1}$ given by (50), we also have :

$$
\begin{aligned}
B_{1} & =\left(\partial_{p^{i}}\left[(1+|\bar{p}|)^{d} f^{M}\right] / P^{i}(1+|\bar{p}|)^{d} f^{M}\right)_{L^{2}(\Omega)} \\
& =-\left((1+|\bar{p}|)^{d} f^{M} / \partial_{p^{i}}\left(P^{i}\right)(1+|\bar{p}|)^{d} f^{M}+P^{i} \partial_{p^{i}}\left[(1+|\bar{p}|)^{d} f^{M}\right]\right)_{L^{2}(\Omega)} .
\end{aligned}
$$

Using the symmetry and bilinearity of the scalar product to the second member of the previous inequality, one has :

$$
B_{1}=-\frac{1}{2}\left(\partial_{p^{i}} P^{i}\left[(1+|\bar{p}|)^{d} f^{M}\right] /(1+|\bar{p}|)^{d} f^{M}\right)_{L^{2}(\Omega)} .
$$


According to lemma $8, \partial_{p^{i}}\left(P^{i}\right)$ is bounded. Hence,

$$
B_{1} \leq \frac{1}{2}\left|\partial_{p^{i}} P^{i}\right|\left\|(1+|\bar{p}|)^{d} f^{M}\right\|_{L^{2}(\Omega)}^{2} \leq C(T, e,|F|)\left\|(1+|\bar{p}|)^{d} f^{M}\right\|_{L^{2}(\Omega)}^{2} .
$$

For the last term $B_{2}$ in (50), we have $\partial_{p^{i}}\left[(1+|\bar{p}|)^{d}\right]=\frac{p^{i} d(1+|\bar{p}|)^{d}}{|\bar{p}|(1+|\bar{p}|)}$, which imply that :

$$
B_{2} \leq\left|\frac{P^{i} p^{i} d(1+|\bar{p}|)^{d}}{|\bar{p}|(1+|\bar{p}|)}\right|\left\|(1+|\bar{p}|)^{d} f^{M}\right\|_{L^{2}(\Omega)}^{2} \leq C(T, e,|F|)\left\|(1+|\bar{p}|)^{d} f^{M}\right\|_{L^{2}(\Omega)}^{2}
$$

(50), (51) and (52) complete the proof of Lemma 9.

Lemma 10. Let $d \in] \frac{N+4}{2}, \infty\left[, f^{M} \in \mathbb{E}_{d}^{m+3}(\Omega), i_{1}=1,2,3\right.$ and $a_{1}=1, \ldots, N-1$. Then :

$$
\begin{aligned}
& \text { (i) }\left|\left((1+|\bar{p}|)^{d+1} \partial_{p^{i_{1}}}\left[P^{i} \frac{\partial f^{M}}{\partial p^{i}}\right] /(1+|\bar{p}|)^{d} \partial_{p^{i_{1}}} f^{M}\right)_{L^{2}(\Omega)}\right| \leq \\
& C(T, e,|F|)\left\{\sum_{i=1}^{3}\left\|(1+|\bar{p}|)^{d+1} \partial_{p^{i}} f^{M}\right\|_{L^{2}(\Omega)}\right\} \times\left\|(1+|\bar{p}|)^{d+1} \partial_{p^{i_{1}}} f^{M}\right\|_{L^{2}(\Omega)} \\
& (2 i)\left|\left((1+|\bar{p}|)^{d+1} \partial_{q^{a_{1}}}\left[P^{i} \frac{\partial f^{M}}{\partial p^{i}}\right] /(1+|\bar{p}|)^{d} \partial_{q^{a_{1}}} f^{M}\right)_{L^{2}(\Omega)}\right| \leq \\
& C(T, e,|F|)\left\{\sum_{i=1}^{3}\left\|(1+|\bar{p}|)^{d+1} \partial_{p^{i}} f^{M}\right\|_{L^{2}(\Omega)}\right\} \times\left\|(1+|\bar{p}|)^{d+1} \partial_{q^{a_{1}}} f^{M}\right\|_{L^{2}(\Omega)} .
\end{aligned}
$$

Démonstration. (i) We have $\partial_{p^{i_{1}}}\left[P^{i} \partial_{p^{i}} f^{M}\right]=\partial_{p^{i_{1}}} P^{i} \partial_{p^{i}} f^{M}+P^{i} \partial_{p^{i_{1}} p^{i}}^{2} f^{M}$, from where

$$
\left|\left((1+|\bar{p}|)^{d+1} \partial_{p^{i_{1}}}\left[P^{i} \partial_{p^{i}} f^{M}\right] /(1+|\bar{p}|)^{d+1} \partial_{p^{i_{1}}} f^{M}\right)_{L^{2}(\Omega)}\right| \leq\left|B_{3}\right|+\left|B_{4}\right|,
$$

with $\left\{\begin{array}{l}B_{3}=\left((1+|\bar{p}|)^{d+1} P^{i} \partial_{p^{i_{1}} f}^{2} f^{M} / \partial_{p^{i_{1}}} f^{M}\right)_{L^{2}(\Omega)} \\ B_{4}=\left((1+|\bar{p}|)^{d+1} \partial_{p^{i_{1}}}\left[P^{i}\right] \partial_{p^{i}} f^{M} /(1+|\bar{p}|)^{d+1} / \partial_{p^{i_{1}}} f^{M}\right)_{L^{2}(\Omega)}\end{array}\right.$

For the term $B_{4}$ in (53), since $\partial_{p^{i_{1}}} P^{i}$ is bounded (Lemma 8 ), we get :

$$
B_{4} \leq C(T, e,|F|) \sum_{i=1}^{3}\left\|(1+|\bar{p}|)^{d+1} \partial_{p^{i}} f^{M}\right\|_{L^{2}(\Omega)} \times\left\|(1+|\bar{p}|)^{d+1} \partial_{p^{i_{1}}} f^{M}\right\|_{L^{2}(\Omega)}^{2} .
$$

Now, for the term $B_{3}$ in (53), we have

$$
\partial_{p^{i}}\left[(1+|\bar{p}|)^{d+1} \partial_{p^{i_{1}}} f^{M}\right]=(1+|\bar{p}|)^{d+1} \partial_{p^{i_{1}} p^{i}}^{2} f^{M}+\partial_{p^{i}}\left[(1+|\bar{p}|)^{d+1}\right] \partial_{p^{i_{1}}} f^{M} .
$$

Thus, $B_{3} \leq\left|B_{3}^{\prime}\right|+\left|B_{3}^{\prime \prime}\right|$, with $B_{3}^{\prime}=\left(P^{i} \partial_{p^{i}}\left[(1+|\bar{p}|)^{d+1} \partial_{p^{i_{1}}} f^{M}\right] /(1+|\bar{p}|)^{d+1} \partial_{p^{i_{1}}} f^{M}\right)_{L^{2}}$ and $B_{3}^{\prime \prime}=\left(P^{i} \partial_{p^{i}}\left[(1+|\bar{p}|)^{d+1}\right] \partial_{p^{i_{1}}} f^{M} /(1+|\bar{p}|)^{d+1} \partial_{p^{i_{1}}} f^{M}\right)_{L^{2}(\Omega)}$. Where, we have on the one hand

$$
\left|B_{3}^{\prime}\right| \leq C(T, e,|F|)\left\|(1+|\bar{p}|)^{d+1} \partial_{p^{i_{1}}} f^{M}\right\|_{L^{2}(\Omega)}^{2},
$$

and on the other hand $\partial_{p^{i}}\left[(1+|\bar{p}|)^{d+1}\right]=\frac{(d+1) p^{i}(1+|\bar{p}|)^{d+1}}{|\bar{p}|(1+|\bar{p}|)}$, which imply that :

$$
\begin{aligned}
\left|B_{3}^{\prime \prime}\right| & \leq\left|\frac{(d+1) P^{i} p^{i}(1+|\bar{p}|)^{d+1}}{|\bar{p}|(1+|\bar{p}|)}\right|\left\|(1+|\bar{p}|)^{d+1} \partial_{p^{i_{1}}} f^{M}\right\|_{L^{2}(\Omega)}^{2} \\
& \leq C(T, e,|F|)\left\|(1+|\bar{p}|)^{d+1} \partial_{p^{i_{1}}} f^{M}\right\|_{L^{2}(\Omega)}^{2} .
\end{aligned}
$$

The inequality (54), (55) and (56) end the proof for (i). 
2i) For the case 2i) We have $\partial_{q^{a_{1}}}\left[P^{i} \partial_{p^{i}} f^{M}\right]=\partial_{q^{a_{1}}} P^{i} \partial_{p^{i}} f^{M}+P^{i} \partial_{q^{a_{1}} p^{i}}^{2} f^{M}$, from where

$$
\left|\left((1+|\bar{p}|)^{d+1} \partial_{q^{a_{1}}}\left[P^{i} \frac{\partial f^{M}}{\partial p^{i}}\right] /(1+|\bar{p}|)^{d} \partial_{q^{a_{1}}} f^{M}\right)_{L^{2}(\Omega)}\right| \leq\left|B_{5}\right|+\left|B_{6}\right| .
$$

with $\left\{\begin{array}{l}B_{5}=\left((1+|\bar{p}|)^{d+1} \partial_{q^{a_{1}}} P^{i} \partial_{p^{i}} f^{M} /(1+|\bar{p}|)^{d} \partial_{q^{a_{1}}} f^{M}\right)_{L^{2}(\Omega)} \\ B_{6}=\left((1+|\bar{p}|)^{d+1} P^{i} \partial_{q^{a_{1}} p^{i}}^{2} f^{M} /(1+|\bar{p}|)^{d} \partial_{q^{a_{1}}} f^{M}\right)_{L^{2}(\Omega)}\end{array}\right.$

In the term $B_{5}$ given by (57), since $\partial_{q^{a_{1}}} P^{i}$ is bounded (Lemma 8 ), we get :

$$
\left|B_{5}\right| \leq C(T, e,|F|)\left\|(1+|\bar{p}|)^{d+1} \partial_{p^{i}} f^{M}\right\|_{L^{2}}\left\|(1+|\bar{p}|)^{d+1} \partial_{q^{a_{1}}} f^{M}\right\|_{L^{2}} .
$$

For the term $B_{6}$ in (57), we have :

$$
\partial_{p^{i}}\left[(1+|\bar{p}|)^{d+1} \partial_{q^{a_{1}}} f^{M}\right]=(1+|\bar{p}|)^{d+1} \partial_{p^{i} q^{a_{1}}}^{2} f^{M}+\partial_{p^{i}}(1+|\bar{p}|)^{d+1} \partial_{q^{a_{1}}} f^{M} .
$$

Thus $\left|B_{6}\right| \leq\left|B_{6}^{\prime}\right|+\left|B_{6}^{\prime \prime}\right|$, with $B_{6}^{\prime}=\left(P^{i} \partial_{p^{i}}\left\{(1+|\bar{p}|)^{d+1} \partial_{q^{a_{1}}} f^{M}\right\} /(1+|\bar{p}|)^{d+1} \partial_{q^{a_{1}}} f^{M}\right)_{L^{2}(\Omega)}$ and $B_{6}^{\prime \prime}=$ $\left(P^{i} \partial_{p^{i}}(1+|\bar{p}|)^{d+1} \partial_{q^{a_{1}}} f^{M} /(1+|\bar{p}|)^{d+1} \partial_{q^{a_{1}}} f^{M}\right)_{L_{2}(\Omega)}$. In which we have on the one hand

$$
\left|B_{6}^{\prime}\right| \leq C(T, e,|F|)\left\|(1+|\bar{p}|)^{d+1} \partial_{q^{a_{1}}} f^{M}\right\|_{L^{2}(\Omega)}^{2},
$$

and the other hand

$$
\begin{aligned}
\left|B_{6}^{\prime \prime}\right| & \leq\left|\frac{(d+1)\left|p^{i}\right|(1+|\bar{p}|)^{d+1}}{|\bar{p}|(1+|\bar{p}|)}\right|\left\|(1+|\bar{p}|)^{d+1} \partial_{q^{a_{1}}} f^{M}\right\|_{L^{2}(\Omega)} \\
& \leq C(T, e,|F|)\left\|(1+|\bar{p}|)^{d+1} \partial_{q^{a_{1}}} f^{M}\right\|_{L^{2}(\Omega)} .
\end{aligned}
$$

The inequality (58), (59) and (60) end the proof for (2i)

Lemma 11. Let $d \in] \frac{N+4}{2}, \infty\left[, f^{M} \in \mathbb{E}_{d}^{m+3}(\Omega)\right.$ and $\beta \in \mathbb{N}^{N+2},|\beta| \leq m+2$ if

$$
\begin{gathered}
\left((1+|\bar{p}|)^{d+|\beta|} \partial_{(\bar{p}, \tilde{q})}^{\beta}\left[P^{i} \frac{\partial f^{M}}{\partial p^{i}}\right] /(1+|\bar{p}|)^{d+|\beta|} \partial_{(\bar{p}, \tilde{q})}^{\beta} f^{M}\right)_{L^{2}(\Omega)} \leq \\
C(T, e,|F|)\left(\sum_{|\alpha| \leq|\beta|}\left\|(1+|\bar{p}|)^{d+|\alpha|} \partial_{(\bar{p}, \tilde{q})}^{\alpha} f^{M}\right\|_{L^{2}(\Omega)}\right)\left\|(1+|\bar{p}|)^{d+|\beta|} \partial_{(\bar{p}, \tilde{q})}^{\beta} f^{M}\right\|_{L^{2}(\Omega)}
\end{gathered}
$$

then, $\forall \beta^{\prime} \in \mathbb{N}^{N+3},\left|\beta^{\prime}\right|=1$, we have :

$$
\begin{gathered}
\left((1+|\bar{p}|)^{d+|\beta|+1} \partial_{(\bar{p}, \tilde{q})}^{\beta+\beta^{\prime}}\left[P^{i} \frac{\partial f^{M}}{\partial p^{i}}\right] /(1+|\bar{p}|)^{d+|\beta|+1} \partial_{(\bar{p}, \tilde{q})}^{\beta+\beta^{\prime}} f^{M}\right)_{L^{2}(\Omega)} \leq \\
C(T, e,|F|)\left(\sum_{|\alpha| \leq\left|\beta+\beta^{\prime}\right|}\left\|(1+|\bar{p}|)^{d+|\alpha|} \partial_{(\bar{p}, \tilde{q})}^{\alpha} f^{M}\right\|_{L^{2}(\Omega)}\right)\left\|(1+|\bar{p}|)^{d+|\beta|+1} \partial_{(\bar{p}, \tilde{q})}^{\beta+\beta^{\prime}} f^{M}\right\|_{L^{2}(\Omega)}
\end{gathered}
$$

Démonstration. We set $\lambda=\beta+\beta^{\prime}$. According to the Leibniz formula, we have :

$$
\partial_{(\bar{p}, \tilde{q})}^{\beta+\beta^{\prime}}\left[P^{i} \frac{\partial f^{M}}{\partial p^{i}}\right]=\sum_{k \leq \lambda} C_{\lambda}^{k} \partial_{(\bar{p}, \tilde{q})}^{k} P^{i} \partial_{(\bar{p}, \tilde{q})}^{\lambda-k}\left(\frac{\partial f^{M}}{\partial p^{i}}\right)
$$


which imply

$$
\left|\left((1+|\bar{p}|)^{d+|\beta|+1} \partial_{(\bar{p}, \tilde{q})}^{\beta+\beta^{\prime}}\left[P^{i} \frac{\partial f^{M}}{\partial p^{i}}\right] /(1+|\bar{p}|)^{d+|\beta|+1} \partial_{(\bar{p}, \tilde{q})}^{\beta+\beta^{\prime}} f^{M}\right)_{L^{2}(\Omega)}\right| \leq\left|K_{1}\right|+\left|K_{2}\right|,
$$

where : $\left\{\begin{array}{l}K_{1}=\left((1+|\bar{p}|)^{d+|\beta|+1} P^{i} \partial_{(\bar{p}, \tilde{q})}^{\beta+\beta^{\prime}}\left(\frac{\partial f^{M}}{\partial p^{i}}\right) /(1+|\bar{p}|)^{d+|\beta|+1} \partial_{(\bar{p}, \tilde{q})}^{\beta+\beta^{\prime}} f^{M}\right)_{L^{2}(\Omega)} \\ K_{2}=\left((1+|\bar{p}|)^{d+|\beta|+1} \sum_{k \leq \lambda,|k| \geq 1} C_{\lambda}^{k} \partial_{(\bar{p}, \tilde{q})}^{k} P^{i} \partial_{(\bar{p}, \tilde{q})}^{\lambda-k}\left(\frac{\partial f^{M}}{\partial p^{i}}\right) /(1+|\bar{p}|)^{d+|\beta|+1} \partial_{(\bar{p}, \tilde{q})}^{\beta+\beta^{\prime}} f^{M}\right)_{L^{2}(\Omega)}\end{array}\right.$

Estimation of $K_{2}$ : According to lemma $8, \partial_{(\bar{p}, \tilde{q})}^{k} \tilde{P}^{i}$ is bounded. Thus,

$$
\begin{aligned}
\left|K_{2}\right| \leq C & \sum_{k \leq \lambda,|k| \geq 1} C_{\lambda}^{k}\left|\left((1+|\bar{p}|)^{d+|\beta|-|k|+2} \partial_{(\bar{p}, \tilde{q})}^{\lambda-k}\left(\frac{\partial f^{M}}{\partial p^{i}}\right) /(1+|\bar{p}|)^{d+|\beta|+1} \partial_{(\bar{p}, \tilde{q})}^{\beta+\beta^{\prime}} f^{M}\right)_{L^{2}(\Omega)}\right|_{k \leq \lambda,|k| \geq 1} C_{\lambda}^{k}\left|\left((1+|\bar{p}|)^{d+|\beta|-|k|+2} \partial_{(\bar{p}, \tilde{q})}^{\lambda+\gamma-k}\left(f^{M}\right) /(1+|\bar{p}|)^{d+|\beta|+1} \partial_{(\bar{p}, \tilde{q})}^{\beta+\beta^{\prime}} f^{M}\right)_{L^{2}(\Omega)}\right|, \\
=C \sum_{k \leq 1} & =
\end{aligned}
$$

with $\gamma \in \mathbb{N}^{3}$ such that $|\gamma|=1$. Hence,

$$
\left|K_{2}\right| \leq C \sum_{k \leq \lambda,|k| \geq 1}\left\|(1+|\bar{p}|)^{d+|\beta|-|k|+2} \partial_{(\bar{p}, \tilde{q})}^{\lambda+\gamma-k} f^{M N}\right\|_{L^{2}(\Omega)}\left\|(1+|\bar{p}|)^{d+|\beta|+1} \partial_{(\bar{p}, \tilde{q})}^{\beta+\beta^{\prime}} f^{M}\right\|_{L^{2}(\Omega)}
$$

Estimation of $K_{1}$ given by (61). We have :

$$
\partial_{p^{i}}\left[(1+|\bar{p}|)^{d+|\beta|+1} \partial_{(\bar{p}, \tilde{q})}^{\beta+\beta^{\prime}} f^{M}\right]=\partial_{p^{i}}\left[(1+|\bar{p}|)^{d+|\beta|+1}\right] \partial_{(\bar{p}, \tilde{q})}^{\beta+\beta^{\prime}} f^{M}+(1+|\bar{p}|)^{d+|\beta|+1} \partial_{(\bar{p}, \tilde{q})}^{\beta+\beta^{\prime}}\left(\frac{\partial f^{M}}{\partial p^{i}}\right) .
$$

from where

$$
\left|K_{1}\right| \leq\left|K_{1}^{\prime}\right|+\left|K_{1}^{\prime \prime}\right|
$$

with $\left\{\begin{array}{l}K_{1}^{\prime}=\left(P^{i} \partial_{p^{i}}\left[(1+|\bar{p}|)^{d+|\beta|+1} \partial_{(\bar{p}, \tilde{q})}^{\beta+\beta^{\prime}} f^{M}\right] /(1+|\bar{p}|)^{d+|\beta|+1} \partial_{(\bar{p}, \tilde{q})}^{\beta+\beta^{\prime}} f^{M}\right)_{L^{2}(\Omega)} \\ K_{1}^{\prime \prime}=\left(P^{i} \partial_{p^{i}}\left[(1+|\bar{p}|)^{d+|\beta|+1}\right] \partial_{(\bar{p}, \tilde{q})}^{\beta+\beta^{\prime}} f^{M} /(1+|\bar{p}|)^{d+|\beta|+1} \partial_{(\bar{p}, \tilde{q})}^{\beta+\beta^{\prime}} f^{M}\right)_{L^{2}(\Omega)}\end{array}\right.$

Since $\tilde{P}^{i}$ is bounded, one has :

$$
K_{1}^{\prime} \leq C(T, e,|F|)\left\|(1+|\bar{p}|)^{d+|\beta|+1} \partial_{(\bar{p}, \tilde{q})}^{\beta+\beta^{\prime}} f^{M}\right\|_{L^{2}(\Omega)}^{2} .
$$

furthermore, $\partial_{p^{i}}\left[(1+|\bar{p}|)^{d+|\beta|+1}\right]=\frac{(d+|\beta|+1) p^{i}(1+|\bar{p}|)^{d+|\beta|+1}}{|\bar{p}|(1+|\bar{p}|)}$, imply that :

$$
K_{1}^{\prime \prime} \leq C(T, e,|F|)\left\|(1+|\bar{p}|)^{d+|\beta|+1} \partial_{(\bar{p}, \tilde{q})}^{\beta+\beta^{\prime}} f^{M}\right\|_{L^{2}(\Omega)}^{2} .
$$

With (63), (64) and (65), we get :

$$
K_{1} \leq C(T, e,|F|)\left\|(1+|\bar{p}|)^{d+|\beta|+1} \partial_{(\bar{p}, \tilde{q})}^{\beta+\beta^{\prime}} f^{M}\right\|_{L^{2}(\Omega)}^{2} .
$$

The inquality (61), (62) and (66) complete the proof of lemma 11 . 
Proposition 5. Let $d \in] \frac{N+4}{2}, \infty\left[, f^{M} \in \mathbb{E}_{d}^{m+3}(\Omega), \alpha, \beta \in \mathbb{N}^{N+2},|\alpha| \leq|\beta| \leq m+3\right.$, then

$$
\begin{gathered}
\left|\left((1+|\bar{p}|)^{d+|\beta|} \partial_{(\bar{p}, \tilde{q})}^{\beta}\left[Q^{I} \partial_{q^{I}} f^{M}\right] /(1+|\bar{p}|)^{d+|\beta|} \partial_{(\bar{p}, \tilde{q})}^{\beta} f^{M}\right)_{L^{2}(\Omega)}\right| \leq \\
C(T, e,|F|)\left(\sum_{|\alpha| \leq|\beta|}\left\|(1+|\bar{p}|)^{d+|\alpha|} \partial_{(\bar{p}, \tilde{q})}^{\alpha} f^{M}\right\|_{L^{2}(\Omega)}\right)\left\|(1+|\bar{p}|)^{d+|\beta|} \partial_{(\bar{p}, \tilde{q})}^{\beta} f^{M}\right\|_{L^{2}(\Omega)}
\end{gathered}
$$

where : (/) stands for the scalar product on $L^{2}(\Omega)$.

Démonstration. The proof of this proposition is similar to that of proposition 4,

Proposition 6. Let $d \in] \frac{N+4}{2}, \infty\left[, f^{M} \in \mathbb{E}_{d}^{m+3}(\Omega), \tilde{f} \in \mathbb{E}_{d, \delta}^{m+3}(\Omega), \beta \in \mathbb{N}^{N+2},|\beta| \leq m+3\right.$ and $T>0$. We have :

$$
\left\|(1+|\bar{p}|)^{d+|\beta|} \partial_{(\bar{p}, \tilde{q})}^{\beta} f^{M}\right\|_{L^{2}(\Omega)} \leq C(T, e,|F|)
$$

Démonstration. Since $\tilde{f} \in \mathbb{E}_{d, \delta}^{m+3}(0, T, \Omega), f^{M} \in \mathbb{E}_{d}^{m+3}(\Omega)$ and using theorem 2 , we have $\frac{1}{p^{0}} \mathcal{L}(\tilde{f}, \tilde{f}) \in \mathbb{E}_{d}^{m+3}(\Omega)$ and $(1+|\bar{p}|)^{d+|\beta|} \partial_{(\bar{p}, \tilde{q})}^{\beta} f^{M} \in L^{2}(\Omega)$, for $\beta \in \mathbb{N}^{m+3}$ such that $|\beta| \leq m+3$. From (49), using the bilinearity of the scalar product, we have :

$$
\begin{gathered}
\left((1+|\bar{p}|)^{d+|\beta|} \partial_{(\bar{p}, \tilde{q})}^{\beta}\left[\partial_{t} f^{M}\right] /(1+|\bar{p}|)^{d+|\beta|} \partial_{(\bar{p}, \tilde{q})}^{\beta} f^{M}\right)_{L^{2}(\Omega)}+ \\
\left((1+|\bar{p}|)^{d+|\beta|} \partial_{(\bar{p}, \tilde{q})}^{\beta}\left[P^{i} \partial_{p^{i}} f^{M}\right] /(1+|\bar{p}|)^{d+|\beta|} \partial_{(\bar{p}, \tilde{q})}^{\beta} f^{M}\right)_{L^{2}(\Omega)}+ \\
\left((1+|\bar{p}|)^{d+\beta \mid} \partial_{(\bar{p}, \tilde{q})}^{\beta}\left[Q^{I} \partial_{q^{I}} f^{M}\right] /(1+|\bar{p}|)^{d+|\beta|} \partial_{(\bar{p}, \tilde{q})}^{\beta} f^{M}\right)_{L^{2}(\Omega)} \\
=\left((1+|\bar{p}|)^{d+|\beta|} \partial_{(\bar{p}, \tilde{q})}^{\beta}\left[\frac{1}{p^{0}} \mathcal{L}(\tilde{f}, \tilde{f})\right] /(1+|\bar{p}|)^{d+|\beta|} \partial_{(\bar{p}, \tilde{q})}^{\beta} f^{M}\right)_{L^{2}(\Omega)} .
\end{gathered}
$$

Given that $(1+|\bar{p}|)^{d+|\beta|}$ does not depend on $t$, we have :

$$
(1+|\bar{p}|)^{d+|\beta|} \partial_{(\bar{p}, \tilde{q})}^{\beta}\left[\partial_{t} f^{M}\right]=\partial_{t}(1+|\bar{p}|)^{d+\beta \mid} \partial_{(\bar{p}, \tilde{q})}^{\beta}\left[f^{M}\right] .
$$

It comes that :

$$
\begin{gathered}
\left((1+|\bar{p}|)^{d+|\beta|} \partial_{(\bar{p}, \tilde{q})}^{\beta}\left[\partial_{t} f^{M}\right] /(1+|\bar{p}|)^{d+|\beta|} \partial_{(\bar{p}, \tilde{q})}^{\beta} f^{M}\right)_{L^{2}(\Omega)}= \\
\left(\partial_{t}(1+|\bar{p}|)^{d+|\beta|} \partial_{(\bar{p}, \tilde{q})}^{\beta} f^{M} /(1+|\bar{p}|)^{d+|\beta|} \partial_{(\bar{p}, \tilde{q})}^{\beta} f^{M}\right)_{L^{2}(\Omega)}=\frac{1}{2} \frac{d}{d t}\left\|(1+|\bar{p}|)^{d+|\beta|} \partial_{(\bar{p}, \tilde{q})}^{\beta} f^{M}\right\|_{L^{2}(\Omega)}^{2}
\end{gathered}
$$

From (67), we then have :

$$
\begin{aligned}
& \frac{1}{2} \frac{d}{d t}\left\|(1+|\bar{p}|)^{d+|\beta|} \partial_{(\bar{p}, \tilde{q})}^{\beta}\left[f^{M}\right]\right\|_{L^{2}(\Omega)}^{2} \\
+ & \left((1+|\bar{p}|)^{d+|\beta|} \partial_{(\bar{p}, \tilde{q})}^{\beta}\left[P^{i} \partial_{p^{i}} f^{M}\right] /(1+|\bar{p}|)^{d+|\beta|} \partial_{(\bar{p}, \tilde{q})}^{\beta} f^{N}\right)_{L^{2}(\Omega)} \\
+ & \left((1+|\bar{p}|)^{d+|\beta|} \partial_{(\bar{p}, \tilde{q})}^{\beta}\left[Q^{I} \partial_{q^{I}} f^{M}\right] /(1+|\bar{p}|)^{d+|\beta|} \partial_{(\bar{p}, \tilde{q})}^{\beta} f^{M}\right)_{L^{2}(\Omega)} \\
=( & \left.(1+|\bar{p}|)^{d+|\beta|} \partial_{(\bar{p}, \tilde{q})}^{\beta}\left[\frac{1}{p^{0}} \mathcal{L}(\tilde{f}, \tilde{f})\right] /(1+|\bar{p}|)^{d+|\beta|} \partial_{(\bar{p}, \tilde{q})}^{\beta} f^{M}\right)_{L^{2}(\Omega)} .
\end{aligned}
$$


Furthermore, according to the Cauchy-Schwartz's inequality, we have :

$$
\begin{aligned}
& \left|\left((1+|\bar{p}|)^{d+|\beta|} \partial_{(\bar{p}, \tilde{q})}^{\beta}\left[\frac{1}{p^{0}} \mathcal{L}(\tilde{f}, \tilde{f})\right] /(1+|\bar{p}|)^{d+|\beta|} \partial_{(\bar{p}, \tilde{q})}^{\beta} f^{M}\right)_{L^{2}(\Omega)}\right| \\
\leq & \left\|(1+|\bar{p}|)^{d+|\beta|} \partial_{(\bar{p}, \tilde{q})}^{\beta}\left[\frac{1}{p^{0}} \mathcal{L}(\tilde{f}, \tilde{f})\right]\right\|_{L^{2}(\Omega)} \times\left\|(1+|\bar{p}|)^{d+|\beta|} \partial_{(\bar{p}, \tilde{q})}^{\beta} f^{M}\right\|_{L^{2}(\Omega)} \\
\leq & \left\|\frac{1}{p^{0}} \mathcal{L}(\tilde{f}, \tilde{f})\right\|_{E_{d}^{m+3}(\Omega)} \times\left\|(1+|\bar{p}|)^{d+|\beta|} \partial_{(\bar{p}, \tilde{q})}^{\beta} f^{M}\right\|_{L^{2}(\Omega)} .
\end{aligned}
$$

So, from (68) we have :

$$
\begin{aligned}
\frac{1}{2} \frac{d}{d t} \|(1+ & |\bar{p}|)^{d+\beta \mid} \partial_{(\bar{p}, \tilde{q})}^{\beta}\left[f^{M}\right] \|_{L^{2}(\Omega)}^{2} \leq \\
& -\left((1+|\bar{p}|)^{d+|\beta|} \partial_{(\bar{p}, \tilde{q})}^{\beta}\left[P^{i} \partial_{p^{i}} f^{M}\right] /(1+|\bar{p}|)^{d+\beta \mid} \partial_{(\bar{p}, \tilde{q})}^{\beta} f^{M}\right)_{L^{2}(\Omega)} \\
& -\left((1+|\bar{p}|)^{d+|\beta|} \partial_{(\bar{p}, \tilde{q})}^{\beta}\left[Q^{I} \partial_{q^{I}} f^{M}\right] /(1+|\bar{p}|)^{d+\beta \mid} \partial_{(\bar{p}, \tilde{q})}^{\beta} f^{M}\right)_{L^{2}(\Omega)}+ \\
& \left\|\frac{1}{p^{0}} \mathcal{L}(\tilde{f}, \tilde{f})\right\|_{E_{d}^{m+3}(\Omega)} \times\left\|(1+|\bar{p}|)^{d+|\beta|} \partial_{(\bar{p}, \tilde{q})}^{\beta} f^{M}\right\|_{L^{2}(\Omega)}
\end{aligned}
$$

Introducing propositions 4 and 5 in (69), we get :

$$
\begin{gathered}
\frac{1}{2} \frac{d}{d t}\left\|(1+|\bar{p}|)^{d+\beta \mid} \partial_{(\bar{p}, \tilde{q})}^{\beta}\left[f^{M}\right]\right\|_{L^{2}(\Omega)}^{2} \\
\leq C(T, e,|F|)\left(\sum_{|\alpha| \leq|\beta|}\left\|(1+|\bar{p}|)^{d+|\alpha|} \partial_{(\bar{p}, \tilde{q})}^{\alpha} f^{M}\right\|_{L^{2}}\right)\left\|(1+|\bar{p}|)^{d+\beta \mid} \partial_{(\bar{p}, \tilde{q})}^{\beta} f^{M}\right\|_{L^{2}(\Omega)} \\
+\left\|\frac{1}{p^{0}} \mathcal{L}(\tilde{f}, \tilde{f})\right\|_{\mathbb{E}_{d}^{m+3}(\Omega)} \times\left\|(1+|\bar{p}|)^{d+|\beta|} \partial_{(\bar{p}, \tilde{q})}^{\beta} f^{M}\right\|_{L^{2}(\Omega)} .
\end{gathered}
$$

Knowing that :

$$
\frac{1}{2} \frac{d}{d t}\left\|(1+|\bar{p}|)^{d+\beta \mid} \partial_{(\bar{p}, \tilde{q})}^{\beta} f^{M}\right\|_{L^{2}(\Omega)}^{2}=\left\|(1+|\bar{p}|)^{d+\beta \mid} \partial_{(\bar{p}, \tilde{q})}^{\beta} f^{M}\right\|_{L^{2}(\Omega)} \frac{d}{d t}\left\|(1+|\bar{p}|)^{d+\beta \mid} \partial_{(\bar{p}, \tilde{q})}^{\beta} f^{M}\right\|_{L^{2}(\Omega)},
$$

dividing (70) by $\left\|(1+|\bar{p}|)^{d+|\beta|} \partial_{(\bar{p}, \tilde{q})}^{\beta} f^{M}\right\|_{L^{2}(\Omega)}$, we have :

$$
\begin{gathered}
\frac{d}{d t}\left\|(1+|\bar{p}|)^{d+\beta \mid} \partial_{(\bar{p}, \tilde{q})}^{\beta} f^{M}\right\|_{L^{2}(\Omega)} \\
\leq C(T, e,|F|)\left(\sum_{|\alpha| \leq|\beta|}\left\|(1+|\bar{p}|)^{d+\alpha \mid} \partial_{(\bar{p}, \tilde{q})}^{\alpha} f^{M}\right\|_{L^{2}(\Omega)}\right)+\left\|\frac{1}{p^{0}} \mathcal{L}(\tilde{f}, \tilde{f})\right\|_{E_{d}^{m+3}(\Omega)} .
\end{gathered}
$$

Summing (71) over $|\beta|=0,1,2, \ldots, m+3$, we get :

$$
\begin{gathered}
\frac{d}{d t}\left(\sum_{|\beta| \leq m+3}\left\|(1+|\bar{p}|)^{d+|\beta|} \partial_{(\bar{p}, \tilde{q})}^{\beta} f^{M}\right\|_{L^{2}(\Omega)}\right) \leq \\
C(T, e,|F|)\left(\sum_{|\beta| \leq m+3}\left\|(1+|\bar{p}|)^{d+|\beta|} \partial_{(\bar{p}, \tilde{q})}^{\alpha} f^{M}\right\|_{L^{2}(\Omega)}\right)+C\left\|\frac{1}{p^{0}} \mathcal{L}(\tilde{f}, \tilde{f})\right\|_{\mathbb{E}_{d}^{m+3}(\Omega)} .
\end{gathered}
$$


By the Gronwall's lemma, using theorem 2, the above inequality yields :

$$
\sum_{|\beta| \leq m+3}\left\|(1+|\bar{p}|)^{d+|\beta|} \partial_{(\bar{p}, \tilde{q})}^{\beta} f^{M}\right\|_{L^{2}} \leq e^{C T}\left[\left\|f_{0}\right\|_{\mathbb{E}_{d}^{m+3}(\Omega)}+C T\|\tilde{f}\|_{\mathbb{E}_{d}^{m+3}(\Omega)}^{2}\right] .
$$

Since $\tilde{f}, f_{0} \in \mathbb{E}_{d, \delta}^{m+3}(\Omega)$, we conclude that $\left\|(1+|\bar{p}|)^{d+|\beta|} \partial_{(\bar{p}, \tilde{q})}^{\beta} f^{M}\right\|_{L^{2}(\Omega)} \leq C(T, e,|F|)$.

Theorem 3. Let $f_{0} \in E_{d, \delta}^{m+3}(\Omega), \tilde{f} \in E_{d, \delta}^{m+3}(\Omega)$ be given. Then the linearized Boltzmann equation (45) has in $\mathbb{E}_{d}^{m+3}(\Omega)$ a local unique and bounded solution.

Démonstration. The proof of this theorem will be done into two steps.

Existence : According to the proposition 6, the sequence $\left(f^{M}\right)_{M}$ is bounded in $\mathbb{E}_{d}^{m+3}(\Omega)$ which is a Hilbert space. So $\left(f^{M}\right)_{M}$ admits a subsequence $\left(f^{M_{k}}\right)_{M_{k}}$ which converges weakly to $f \in \mathbb{E}_{d}^{m+3}(\Omega)$, the solution of the linearized Boltzmann equation (45). From (47), we have :

$$
\left(\partial_{t} f^{M_{k}} / v_{k}\right)+\left(P^{i} \partial_{p^{i}} f^{M_{k}} / v_{k}\right)+\left(Q^{I} \partial_{q^{I}} f^{M_{k}} / v_{k}\right)=\left(\frac{1}{p^{0}} \mathcal{L}(\tilde{f}, \tilde{f}) / v_{k}\right) .
$$

At the limit when $k \longrightarrow+\infty$, we obtain $\partial_{t} f+P^{i} \partial_{p^{i}} f+Q^{I} \partial_{q^{I}} f=\frac{1}{p^{0}} \mathcal{L}(\tilde{f}, \tilde{f})$. Hence $f$ is a solution of the Boltzmann equation (45) satisfying the condition $f(0)=f_{0}$.

Uniqueness : We assume that, there is another solution $f^{\prime}$ of (45) with the same initial data $f_{0}$. By setting $g=f-f^{\prime}, g$ is the solution of the following cauchy problem

$$
\left\{\begin{array}{l}
\frac{\partial g}{\partial t}+P^{i} \frac{\partial g}{\partial p^{i}}+Q^{I} \frac{\partial g}{\partial q^{I}}=0 \\
g(0)=0
\end{array}\right.
$$

Next, proceeding as in the proof of the proposition 6, we show that (74) admits $g=0$ as a unique solution. Thus $f=f^{\prime}$

Theorem 4. Let $f_{0} \in E_{d, \delta}^{m+3}(\Omega)$ be given. Then the Boltzmann equation (43) has in $E_{d, \delta}^{m+3}(\Omega)$ a local unique solution $f$ such that $f(0, \bar{p}, \tilde{q})=f_{0}(\bar{p}, \tilde{q})$.

Démonstration. We consider the application

$$
\phi: E_{d}^{m+3} \longrightarrow E_{d}^{m+3}(\Omega) ; \tilde{f} \longmapsto \phi(\tilde{f})=f
$$

where $f$ is the unique solution of (43). The proof of this theorem will be divided into two steps.

1) We will show that one can choose $f_{0} \in E_{d, \delta}^{m+3}(\Omega)$ and $T>0$ such that $\phi$ sends $E_{d, \delta}^{m+3}(\Omega)$ in itself.

In fact, this is to show that one can choose $f_{0} \in E_{d, \delta}^{m+3}(\Omega)$ and $T>0$ such that

$$
\|\tilde{f}\|_{\mathbb{E}_{d}^{m+3}(\Omega)} \leq \delta \quad \Rightarrow \quad\|f\|_{\mathbb{E}_{d}^{m+3}(\Omega)} \leq \delta
$$

By introducing (73) in (72), one has :

$$
\begin{gathered}
\frac{d}{d t}\left(\sum_{|\beta| \leq m+3}\left\|(1+|\bar{p}|)^{d+|\beta|} \partial_{(\bar{p}, \tilde{q})}^{\beta} f^{M}\right\|_{L^{2}(\Omega)}\right) \leq \\
C(T, e,|F|)\left(\left\|f_{0}\right\|_{\mathbb{E}_{d}^{m+3}(\Omega)}+C T\|\tilde{f}\|_{\mathbb{E}_{d}^{m+3}(\Omega)}^{2}+\left\|\frac{1}{p^{0}} \mathcal{L}(\tilde{f}, \tilde{f})\right\|_{\mathbb{E}_{d}^{m+3}(\Omega)}\right) .
\end{gathered}
$$


Integrating this inequality on $[0, t], 0 \leq t<T$, we obtain using $\left\|\frac{1}{p^{0}} \mathcal{L}(\tilde{f}, \tilde{f})\right\|_{\mathbb{E}_{d}^{m+3}} \leq \delta^{2}$ :

$$
\begin{gathered}
\sum_{|\beta| \leq m+3}\left\|(1+|\bar{p}|)^{d+|\beta|} \partial_{(\bar{p}, \tilde{q})}^{\beta} f^{M}\right\|_{L^{2}} \leq \sum_{|\beta| \leq m+3}\left\|(1+|\bar{p}|)^{d+|\beta|} \partial_{(\bar{p}, \tilde{q})}^{\beta} f^{M}(0)\right\|_{L^{2}}+ \\
C(T, e,|F|)\left(\left\|f_{0}\right\|_{\mathbb{E}_{d}^{m+3}(\Omega)} T+C T^{2} \delta^{2}+\delta^{2} T\right) .
\end{gathered}
$$

But, we have

$$
\left\|f^{M}\right\|_{\mathbb{E}_{d}^{m+3}} \leq\left\|f_{0}\right\|_{\mathbb{E}_{d}^{m+3}}+C(T, e,|F|)\left(\left\|f_{0}\right\|_{\mathbb{E}_{d}^{m+3}(\Omega)} T+C T^{2} \delta^{2}+\delta^{2} T\right)
$$

Since $\delta>0$ is given, if we take $f_{0} \in \mathbb{E}_{d, \delta}^{m+3}(\Omega)$ and $T>0$ such that :

$$
\left\|f_{0}\right\|_{\mathbb{E}_{d}^{m+3}(\Omega)} \leq \frac{\delta}{2} \text { and } C(T, e,|F|)\left(\left\|f_{0}\right\|_{\mathbb{E}_{d}^{m+3}(\Omega)} T+C T^{2} \delta^{2}+\delta^{2} T\right) \leq \frac{\delta}{2}
$$

(75) imply $\left\|f^{M}\right\|_{\mathbb{E}_{d}^{m+3}(\Omega)} \leq \delta$. Since the sequence $\left(f^{M}\right)_{M}$ converges weak star to $f$ in $\mathbb{E}_{d}^{m+3}(\Omega)$, we have $\|f\|_{\mathbb{E}_{d}^{m+3}(\Omega)} \leq \delta$. This shows that $\|\tilde{f}\|_{\mathbb{E}_{d}^{m+3}(\Omega)} \leq \delta \Rightarrow\|f\|_{\mathbb{E}_{d}^{m+3}(\Omega)} \leq \delta$.

\section{2)We show that $\phi$ is a contraction in the space $\mathbb{E}_{d, \delta}^{m+3}(\Omega)$.}

Suppose that $\phi\left(\tilde{f}_{j}\right)=f_{j}, j=1,2$ where

$$
\tilde{f}_{j}(0)=f_{0} \text { and }\left\|\tilde{f}_{j}\right\|_{\mathbb{E}_{d}^{m+3}(\Omega)} \leq \delta, j=1,2
$$

show that

$$
\left\|f_{1}-f_{2}\right\|_{\mathbb{E}_{d}^{m+3}} \leq T C(\tilde{F}, T)\left\|\tilde{f}_{1}-\tilde{f}_{2}\right\|_{\mathbb{E}_{d}^{m+3}(\Omega)}
$$

Setting $G=f_{1}-f_{2}$ and $\tilde{G}=\tilde{f}_{1}-\tilde{f}_{2}$ then, $G \in \mathbb{E}_{d}^{m+3}(\Omega)$ is a solution of the following Cauchy problem

$$
\left\{\begin{array}{l}
\frac{\partial G}{\partial t}+P^{i} \partial_{p^{i}} G+Q^{I} \partial_{q^{I}} G=\frac{1}{p^{0}} \mathcal{L}\left(\tilde{f}_{1}, \tilde{G}\right)+\frac{1}{p^{0}} \mathcal{L}\left(\tilde{G}, \tilde{f}_{2}\right) \\
G(0)=0
\end{array}\right.
$$

(72) gives

$$
\begin{gathered}
\frac{d}{d t}\left\|(1+|\bar{p}|)^{d+|\beta|} D_{(\bar{p}, \tilde{q})}^{\beta} G(t)\right\|_{L^{2}(\Omega)} \leq \\
C\left\|(1+|\bar{p}|)^{d+|\beta|} D_{(\bar{p}, \tilde{q})}^{\beta} G(t)\right\|_{L^{2}(\Omega)}+\left\|(1+|\bar{p}|)^{d+|\beta|} D_{(\bar{p}, \tilde{q})}^{\beta}\left(\frac{1}{p^{0}} Q\left(\tilde{f}_{1}, \tilde{G}\right)+\frac{1}{p^{0}} Q\left(\tilde{G}, \tilde{f}_{1}\right)\right)\right\|_{L^{2}(\Omega)} .
\end{gathered}
$$

Integrating on $[0, t]$ for $t \in[0, T$ and applying Gronwall's lemma, knowing that $G(0)=0$, we obtain :

$$
\begin{gathered}
\left\|(1+|\bar{p}|)^{d+|\beta|} D_{(\bar{p}, \tilde{q})}^{\beta} G(t)\right\|_{L^{2}(\Omega)} \leq \\
C(T, e,|F|) \int_{0}^{t}\left\|(1+|\bar{p}|)^{d+\beta \mid} D_{(\bar{p}, \tilde{q})}^{\beta}\left(\frac{1}{p^{0}} Q\left(\tilde{f}_{1}, \tilde{G}\right)+\frac{1}{p^{0}} Q\left(\tilde{G}, \tilde{f}_{1}\right)\right)\right\|_{L^{2}(\Omega)} d \tau .
\end{gathered}
$$

Then, taking the supremum in (77), for $t$, we get :

$$
\|G(t)\|_{E_{d}^{m+3}(\Omega)} \leq C(T, e,|F|) T\|\tilde{G}(t)\|_{\mathbb{E}_{d}^{m+3}(\Omega)}
$$

According to (78), $\phi$ is a contracting map if $T>0$ is chosen such that $T \times C(T, e,|F|)<1$. So, by the Banach-Picard's theorem, $\phi$ has a unique fixed point $f$ solution of the Boltzmann equation (43) 
Remark 4. The only explicit dependence of $C$ with respect to $T$ is given in the proof of lemma 8 where we have (in line 8 of this proof) $|\bar{p}(t)| \leq\left(|\bar{p}(0)|+C_{1} T\right) e^{C T}$.

The other dependence of $C$ with respect to $T$ comes through the one's of the components of the YangMills field $F$ and the components of the Yang-Mills potential A, which are given continuous functions of time $t$. Since $[0, T]$ is a compact set, the constant $C$ can be taken absolutely with respect to $T$. So the quantity $T \times C(T, e,|F|)$ tends to zero with $T$. We can then choose $T$ such that $T C<1$.

Conclusion. We have obtained a local in time regular solution for the relativistic Boltzmann equation in Yang-Mills field, taking as background the Minkowski space-time. In future work, we will try to generalize this result in the Robertzon-Walker-space-time which is a curved Lorentzian manifold.

\section{Bibliographie}

[1] R. D. Ayissi, N. Noutchegueme, R. M. Etoua, and H. P. Mbeutchagna, Viscosity solutions for the one-body Liouville equation in Yang-Mills charged Bianchi Models with Non-Zero mass, Lett. Math. Phys., 105 (2015), $1289-1299$.

[2] R. D. Ayissi, N. Noutchegueme, The Faedo-Galerkin method for the relativistic Boltzmann equation in Bianchi type 1 space-time, Comm. Math. Appl., 4 (2013), no. 2, 93-118.

[3] C. Cercignani, Rarefied gas dynamics, From basic concepts to actual calculations, Cambridge University Press, Cambridge 2000.

[4] Y. Choquet-Bruyat, General relativity and the Einstein equations, Oxford University, 2009.

[5] Y. Choquet-Bruhat, N. Noutchegueme, Système de Yang-Mills-Vlasov en jauge temporelle, Ann. Inst. Henri Poincaré, 55 (1991), no. 3, 759-787.

[6] Y. Choquet-Bruhat, N. Noutchegueme, Solution globale des équations de Yang-Mills-Vlasov (masse nulle), C. R. Acad. Sc. Paris, 311 (1990), no. 1, 785-789.

[7] Y. Choquet-Bruhat, D. Christodoulou, Elliptic systems in $H_{s, \delta}$ spaces on manifolds which are euclidean at infinity. Acta Mathematica 146(1), 129-150 (1981).

[8] R. T. Glassey, Lectures on Cauchy Problems, Trans. Amer.Math. Soc. 282 (1993).

[9] P. B. Mucha, Global existence of solutions of the Einstein-Boltzmann equation in the flat Roberson-Walker space-time, Comm. Math. Phys., 203 (1999), 107-118.

[10] N. Noutchegueme, D. Dongo and F. E. Djiofack, Global regular solution for the EinsteinMaxwell-Boltzmann-Scalar field system in Bianchi type I space-time, J. A. M., 13 (2017), 7087-7118.

[11] N. Noutchegueme, M. Kenmogne, Regular solutions to the Boltzmann equation on a Robertson-Walker space-time, J. P. M. S., 5 (2015), no. 4, 66-104.

[12] N. Noutchegueme, R. Ayissi, Bianchi type 1 magnetized cosmological models for the Einstein-Boltzmann equation with the cosmological constant, J. Math. Phys., 56 (2015).

[13] N. Noutchegueme, D. Dongo, Global existence of solutions for the Einstein-Boltzmann system in Bianchi type 1 space-time, Class. Quantum Grav., 23 (2006) pp. 2979-3003.

[14] N. Noutchegueme, D. Dongo and E. Takou, Global existence of solutions for the relativistic Boltzmann equation with arbitrarily large initial data on a Bianchi type 1 space-time, Gen. Relat. Grav., 37 (2005), no. 12, $2047-2062$.

[15] N. Noutchegueme, P. Noundjeu, Systeme de Yang-Mills-Vlasov pour des particules avec densité de charge de jauge non-abélienne sur un espace-temps courbe, Ann. Inst. Henri Poincaré, 1 (2000), 385-404. 